\title{
1 Multiple hybridization events punctuate the evolutionary trajectory of
}

2 Malassezia furfur

3

4 Bart Theelen ${ }^{\mathrm{a}, \S}$, Verónica Mixão ${ }^{\mathrm{b}, \mathrm{c}, *,}$, Giuseppe Ianiri ${ }^{\mathrm{d}}$, Joleen Goh Pei Zhen ${ }^{\mathrm{e}}$, Jan

5 Dijksterhuis ${ }^{\mathrm{a}}$, Joseph Heitman ${ }^{\mathrm{f}}$, Thomas L. Dawson Jr. ${ }^{\mathrm{e}, \mathrm{g}}$, Toni Gabaldón ${ }^{\text {b,c,h,\#,+, Teun }}$

6 Boekhout ${ }^{\mathrm{a}, \mathrm{i}, \#,+}$

7

$8 \quad{ }^{a}$ Westerdijk Fungal Biodiversity Institute, Utrecht, The Netherlands

$9{ }^{\mathrm{b}}$ Life Sciences Department, Barcelona Supercomputing Center (BSC), Barcelona,

10 Spain

$11{ }^{\mathrm{c}}$ Mechanisms of Disease Programme, Institute for Research in Biomedicine (IRB),

12 Barcelona, Spain

$13{ }^{\mathrm{d}}$ Department of Agricultural, Environmental and Food Sciences, University of

14 Molise, 86100 Campobasso, Italy

$15{ }^{\mathrm{e}}$ Skin Research Institute of Singapore (SRIS), Agency for Science, Technology, and

16 Research (A*STAR), Singapore

$17{ }^{\mathrm{f}}$ Department of Molecular Genetics and Microbiology, Duke University Medical

18 Center, Durham, North Carolina, United States of America

$19{ }^{\mathrm{g}}$ Center for Cell Death, Injury and Regeneration, Departments of Drug Discovery and

20 Biomedical Sciences and Biochemistry and Molecular Biology, Medical University of

21 South Carolina, Charleston, South Carolina, USA

$22{ }^{\mathrm{h}}$ Catalan Institution for Research and Advanced Studies (ICREA), Barcelona, Spain

$23{ }^{\mathrm{i}}$ Institute for Biodiversity and Ecosystem Dynamics (IBED), University of

24 Amsterdam, The Netherlands 
1 Running head: Hybridization in Malassezia furfur

2

$3 \quad$ \#Address correspondence to Teun Boekhout (t.boekhout@wi.knaw.nl) and Toni

4 Gabaldón (toni.gabaldon@bsc.es)

5

$6 \quad{ }^{*}$ Present address: Bioinformatics Unit, Infectious Diseases Department, National

7 Institute of Health Dr. Ricardo Jorge, Av. Padre Cruz, 1649-016, Lisbon, Portugal

8

$9 \quad \S$ Bart Theelen and Verónica Mixão contributed equally to this work.

$1{ }^{+}$Toni Gabaldón and Teun Boekhout share senior authorship and correspondence.

11

12 Abstract: 255 words

13 Importance: 149 words

14 Text: 7248 words 


\section{Abstract}

Malassezia species are important fungal skin commensals and are part of the

3 normal microbiota of humans and other animals. However, under certain

4 circumstances these fungi can also display a pathogenic behaviour. For example,

$5 \quad$ Malassezia furfur is a common commensal of human skin, and yet is often

6 responsible for skin disorders but also systemic infections. Comparative genomics

7 analysis of $M$. furfur revealed that some isolates have a hybrid origin, similar to

8 several other recently described hybrid fungal pathogens. Because hybrid species

9 exhibit genomic plasticity that can impact phenotypes, we sought to elucidate the genomic evolution and phenotypic characteristics of $M$. furfur hybrids in comparison to their parental lineages. To this end, we performed a comparative genomics analysis between hybrid strains and their presumptive parental lineages, and assessed phenotypic characteristics. Our results provide evidence that at least two distinct hybridization events occurred between the same parental lineages, and that the parental strains may have originally been hybrids themselves. Analysis of the matingtype locus reveals that $M$. furfur has a pseudobipolar mating system, and provides evidence that after sexual liaisons of mating compatible cells, hybridization involved cell-cell fusion leading to a diploid/aneuploid state. This study provides new insights into the evolutionary trajectory of $M$. furfur and contributes with valuable genomic resources for future pathogenicity studies.

\section{Importance}

23 Malassezia furfur is a common commensal member of human/animal microbiota that

24 is also associated with several pathogenic states. Recent studies report involvement of

Malassezia species in Crohn's disease, a type of inflammatory bowel disease, 
1 pancreatic cancer progression, and exacerbation of Cystic fibrosis. A recent genomics

2 analysis of $M$. furfur revealed the existence of hybrid isolates and identified their

3 putative parental lineages. In this study, we explored the genomic and phenotypic

4 features of these hybrids in comparison to their putative parental lineages. Our results

5 revealed the existence of a pseudobipolar mating system in this species and showed

6 evidence for the occurrence of multiple hybridization events in the evolutionary

$7 \quad$ trajectory of $M$. furfur. These findings significantly advance our understanding of the

8 evolution of this commensal microbe and are relevant for future studies exploring the

9 role of hybridization in the adaptation to new niches or environments, including the

10 emergence of pathogenicity.

\section{Introduction (588 words)}

Malassezia are basidiomycetous yeasts that are the dominant fungal component of the healthy human skin microbiome $(1,2)$. Nevertheless, they can also take on pathogenic roles in various skin disorders, and have been implicated in cases of infection associated with several comorbidities (3-12). Non-culture based sequencing methods revealed Malassezia presence in other ecological niches, including insects, nematodes, corals, sponges, deep-sea environments, and soils (1315), suggesting that Malassezia are ecologically diverse. Interestingly, Malassezia are evolutionarily related to fungal plant pathogens, potentially pointing towards a host shift from plants to animals, facilitated by the loss of genes coding for proteins involved in the degradation of complex carbohydrates, and expansion of lipid hydrolases required to break down lipids available on animal skin $(16,17)$. 
1 In Basidiomycetes, species with either a tetrapolar or bipolar mating system have been

2 described $(18,19)$. In a tetrapolar mating system, the pheromone and pheromone-

3 receptor locus (P/R) and the homeodomain locus (HD) are unlinked, whereas in a

4 bipolar mating system both loci are linked and contiguous on the same chromosome

$5 \quad$ which precludes recombination. An intermediate -pseudobipolar- mating system was

6 described in three Malassezia species, namely Malassezia globosa, Malassezia

7 sympodialis, and Malassezia yamatoensis. In this configuration, the P/R and HD

8 mating-type loci are linked on the same chromosome but far enough apart from each

9 other that recombination can still occur $(16,20,21)$. The presence of mating and

meiotic genes suggests that Malassezia are potentially capable of sexual reproduction,

11 but mating remains hitherto unobserved, and the genus is known to propagate

12 asexually through unipolar budding $(16,21,22)$.

Our study focuses on Malassezia furfur, a species isolated from a wide variety of

hosts, from humans (with healthy skin, skin disorders, or bloodstream infections), to a

range of domestic and zoo animals $(12,15,23-26)$. This species presents variable cell

sizes and shapes and different ploidies and karyotypes (27-29). Indeed, previous

studies reported two different karyotypes -one displaying more chromosomes (10-11

vs. 7-8) and a larger genome size ( 14 Mbp vs. $8.5 \mathrm{Mbp})$ than the other- and a high

degree of genetic variation that uncovered a possible hybrid genotype $(28,30,31)$.

These findings were later corroborated in a genomic analysis of members of the

23 CBS7019, CBS7710) with double genome size due to gene duplication that possibly

24 originated from a hybridization event between members of the lineages of strains

CBS7982 and CBS14141 (syn. JPLK23) (16). 
2 The coexistence of genomic material of two diverged lineages in a single cell is

3 generally expected to have lower fitness $(32,33)$. Nevertheless, several genomic

4 mechanisms, such as recombination, often lead to loss of heterozygosity ( $\mathrm{LOH}$ ), thus

5 erasing some of the genomic incompatibilities (34). In such a scenario, organisms

6 carrying highly plastic genomes and unique phenotypes may survive leading to new

7 lineages able to thrive in new environments (34-36). For instance, findings of

8 multiple pathogenic hybrids, such as Candida albicans or the Cryptococcus gattii /

9 Cryptococcus neoformans species complex, has led to the hypothesis that

10 hybridization plays an important role in the emergence of pathogenicity (30, 37-44).

Taking advantage of the existence of known candidate parental haploid lineages, this

study explored genomic and some general phenotypic features of $M$. furfur hybrids to assess the genomic aftermath of hybridization in this species and determine the existence of genomic and phenotypic alterations in these hybrids when compared to their parentals. This study reveals that $M$. furfur has a pseudobipolar mating system and shows evidence that multiple hybridization events punctuated the evolution of the species.

\section{Results and Discussion (4444 words)}

\section{M. furfur genomic patterns reveal the current existence of two hybrid lineages}

Previous AFLP and comparative genomics analyses suggested the existence of $M$.

furfur hybrid strains $(16,31)$. In this study, we further explored the genomic patterns 
1 observations of a hybrid genotype (31), we performed AFLP with two different

2 adaptor and primer set combinations for 22 M. furfur strains (Supplementary Table 1

3 and Materials and Methods for more details). These AFLP analyses confirmed that

4 the banding patterns of three previously identified hybrids (CBS1878, CBS4172,

5 CBS7019) are indeed a combination of those of the proposed parental lineages

6 (Figure 1) (16). Nevertheless, the hybrid strain CBS7019 did not cluster with the

7 other two, suggesting the existence of two putative hybrid clades (Figure 1). In total,

8 the AFLP clustering analysis identified four lineages: P1 (parental 1), which

9 corresponds to the parental CBS7982 lineage with five additional strains; P2, which

corresponds to the parental CBS14141 lineage and four additional strains; H1 (hybrid

11 1), which corresponds to CBS1878, CBS4172, and five additional hybrid strains; and

12 finally, H2, which harbors CBS7019 and three additional hybrid strains (Figure 1).

[Figure 1]

[Table 1]

To confirm the existence of two independent hybridization events, 13 strains (Table

1) comprising representative isolates of all four AFLP-determined lineages were further compared in detail at the genomic level. To this end, we performed the genome assembly of the representative isolate of each of the parental lineages 
1 approach where all the reads are aligned on a single haplotype would not be possible,

2 and hybrid sequencing reads were mapped to a combined reference including the P1

3 and the P2 (CBS14141: 8.3 Mb, 9 scaffolds) genome assemblies (Materials and

4 Methods for more details) to identify the source and sequence of the different sub-

5 genomes of the hybrids (Figure 2a).

6

$7 \quad$ [Table 2]

9 AFLP-determined $\mathrm{H} 1$ and $\mathrm{H} 2$ lineages on average presented $6 \mathrm{SNPs} / \mathrm{kb}$ (all

homozygous given the alignment to a combined reference, Supplementary Table 2), with the majority of them (>85\%) corresponding to P2 for all hybrid strains, thus suggesting that CBS9595 is possibly closer to the actual parent 1 than CBS14141 is to the actual parent 2. Noteworthy, these SNPs were not homogeneously distributed along the genome, but rather formed blocks of variants (see Supplementary Figure 1a and Materials and Methods for more details on block definition). Therefore, based on the assumption that strains originating from the same hybridization event would share the same blocks of variants, we utilized these as signatures of the hybrids' evolutionary past and compared them between the different hybrids to confirm the number of hybridization events. Jaccard metrics revealed a high block overlap between the strains identified by AFLP as $\mathrm{H} 1$ (>84.6\%), and the strains identified by AFLP as $\mathrm{H} 2(>91.8 \%)$, thus confirming the results of the AFLP analysis. It is important to highlight that among the H1 strains, CBS4172 presented the lowest similarity with its peers. While this strain revealed $84.6 \%$ and $84.9 \%$ block overlap with CBS1878 and CBS9365, respectively, the latter two strains had an overlap of 99\%. The block overlap in strains of hybrid lineage $\mathrm{H} 2$ was more homogeneous. The 
1 overlap of the high polymorphic regions between strains of $\mathrm{H} 1$ and $\mathrm{H} 2$ varied

2 between $20.8 \%$ and $23.1 \%$, thus suggesting independent origins. Together, these

3 results confirm that the hybrid lineages resulted from two independent hybridization

4 events between $\mathrm{P} 1$ and $\mathrm{P} 2$. These findings suggest an apparent propensity of $\mathrm{P} 1$ and

$5 \quad \mathrm{P} 2$ to hybridize, and for their hybrids to survive.

6

$7 \quad$ [Figure 2]

9 Genomic divergence and different mitochondria uncover the hybrid origin of P1 and P2

11 Considering the possible ancestry of the highly polymorphic blocks in the two hybrid

12 lineages, we sought to determine their presence in the parental lineages. To this end, the same methodology was followed as for the hybrids (see Materials and Methods), and we verified that indeed, the strains of both P1 and P2 that were not used for genome assembly also harbor highly polymorphic genomic regions. A comparison between these blocks and the ones observed in the hybrid strains showed that the highest overlap between $\mathrm{H} 1$ strains and $\mathrm{P} 1$ and $\mathrm{P} 2$ is $14.6 \%$ and $21 \%$, respectively. Similarly, comparing the regions of high variability in the hybrid lineage $\mathrm{H} 2$ with what was detected in $\mathrm{P} 1$ and $\mathrm{P} 2$ revealed that the highest overlaps were $64 \%$ and $13 \%$, respectively. This is in line with our previous observations in the hybrid genomes and suggests that none of the strains sequenced for the P1 or P2 lineages represents the direct parental strain of the hybrids, but rather their close relatives.

24 The origin of these blocks of high genomic variability was unknown but, as reported by Mixão and Gabaldón (37) for C. albicans, two possible models could explain their 
1 existence: i) continuous admixture between different strains, and ii) a hybrid ancestor

2 that experienced genomic recombination. A key aspect to distinguish these models is

3 the divergence between the reference genome and the observed sequence, which we

4 expect to vary between the different blocks in the first model, and to be similar among

$5 \quad$ them in the second one (37). Therefore, we next assessed the level of divergence

6 between these blocks and the reference genome in both lineages. For P2 strains, we

7 identified a clear single peak of sequence divergence in both CBS8735 and PM315

8 (Figure 2b), suggesting that the genomic material of all the blocks was acquired at a

9 single time-point, which in turns supports the existence of a hybrid ancestor. Our

estimations point to a current haplotype divergence between the strains of parental

lineage $\mathrm{P} 2$ of $4 \%$ to $4.5 \%$ (Supplementary Table 2 ). To determine if any sequenced

strain could be the alternative parent of P2 lineage, we performed a BLASTn search

in the NCBI genome database. The only hit obtained was the CBS14141 (the same

strain we use as the $\mathrm{P} 2$ reference) genome with $95.2 \%$ sequence similarity, a value

consistent with our estimations. This means that the alternative parent of the P2

lineage has not been sequenced thus far.

For the P1 lineage, this analysis was complicated by the limited number of polymorphic blocks (min $78-\max 463$ blocks), and their short size (average $=245$ bp), because a variation in a single polymorphism can have a large impact on the estimation of the sequence divergence. Indeed, despite the observed overlap between the peaks of sequence divergence in the different P1 strains, we also detected multiple sequence divergence peaks (Figure 2c). Therefore, we could not exclude any of the

24 above-mentioned models (i.e., continuous admixture between different strains, or a hybrid ancestor that experienced genomic recombination) and properly estimate the 
1 sequence divergence. Even so, we performed a BLASTn search in the NCBI genome

2 database using the longest blocks ( $>500 \mathrm{bp}$ ), and we verified that their best hits

3 always presented a sequence similarity of $\sim 93 \%$ with the CBS14141 genome (P2

4 lineage), thus suggesting that P1 also has a hybrid origin.

5

6 We next focused on comparing the mitochondrial genome sequences of the different

$7 \quad$ M. furfur lineages. Genomic reads of each parental strain were mapped against

8 mitochondrial genomes of the respective strains. As reference mitochondria for the P2

9 lineages, we selected the CBS14141 mitochondrial sequence available in NCBI (accession number: KY911086.1). As expected, all P2 strains shared the same mitochondrial genome. In the P1 lineage, the publicly available mitochondrial genome of CBS7982 (NCBI accession number: KY911085.1) was used as a reference for mapping sequencing reads from P1 strains. Mapping results revealed the presence of two different mitochondrial sequences in the P1 lineage with the mitochondrial sequence of CBS7982 differing from that of CBS9595, CD866, and CBS9574 (Supplementary Figure 1b). To compare both P1 mitochondrial types, a draft mitochondrial genome assembly with 49kb was generated for CBS9595 (see Materials and Methods). A BLASTn search with this mitochondrial genome against the NCBI nr database revealed that its sequence is equally distant to that of the mitochondrial genomes of CBS7982 (P1 lineage) and CBS14141 (P2 lineage), with a similarity of $93.45 \%$. These results are in line with our observations for the polymorphic regions of the nuclear genome, further providing support that P1 results from the cross of two diverged lineages, i.e. hybridization, and CBS7982 harbors the mitochondrial genome of the alternative parent. A recent study assessing three mitochondrial genomic loci of 43 M. furfur strains, found two rather divergent 
1 mitochondrial clades for samples belonging to the P1 lineage (45), supporting our

2 results and suggesting a wider presence of two different mitochondria among P1

3 strains. When reads of the strains belonging to both hybrid lineages were mapped

4 against the mitochondrial reference genomes, it was observed that all hybrid strains

$5 \quad$ inherited the mitochondria from the P2 lineage.

6

7 Together, these analyses suggest that both parental lineages are not genetically "pure"

8 but are genomic mosaics likely resulting from a hybridization event between two

9 unknown lineages that are approximately $7 \%$ divergent in their mitochondrial genome

10 in the case of P1 (divergence in the nuclear genome could not be confidently

11 estimated), and 4\% divergent in their nuclear genome in the case of P2 (Figure 3).

12 The observed small differences in the genetic mosaics detected in the nuclear

13 genomes in each of the parental lineages suggest that strains in each lineage (P1 and

14 P2) diverged before some of the recombination events occurred, a scenario which implies a non-haploid state of the strains at the time of their respective divergence. Nevertheless, all analyses performed in this study support a haploid state for all of them (Supplementary Figures 2 and 3), indicating independent ploidy reduction.

[Figure 3]

\section{M. furfur hybrid lineages are mostly diploid with some LOH}

Karyotype data from this study and previous publications $(46,47)$ demonstrate

significant chromosomal variation in $M$. furfur with hybrid strains containing

24 additional chromosomal bands when compared to strains from their hypothesized 
1 analysis for H1 strains displayed a DNA content between 1n and 2n suggesting

2 aneuploidy, whereas the DNA-content of both analyzed H2 strains is consistent with

3 assignment as diploids (Supplementary Figure 2b). Based on whole-genome

4 sequencing data analysis, the analyzed strains for both hybrid lineages generally seem

5 of diploid nature (Supplementary Figures 4 and 5), with a few exceptions, such as a

6 putative triplication of a chromosome from P2 in the hybrid strain CBS9365 (H1,

$7 \quad$ Supplementary Figure 4).

9 Considering the non-haploid state of these hybrid strains, we decided to look for LOH events, an important feature to restore genomic stability through the erasure of one of the haplotypes of the recombining region (34). In the case of the $M$. furfur hybrids analyzed in this study, we estimated that LOH covers approximately $20 \%$ of the genome (Supplementary Table 2). This is a low value when compared to other fungal hybrids, such as Candida hybrids, where LOH has been estimated to cover at least $50 \%$ of the genome $(37-40,44)$. Considering that sequence divergence between the parental lineages is higher in M. furfur $(\sim 10 \%)$ than in Candida hybrids ( 4\%) (3740, 44), we hypothesize that the lower occurrence of LOH in M. furfur may be related to a lower number of potential recombining sites. Indeed, a recent study on $C$. neoformans x $C$. gattii hybrids, which present $7 \%$ sequence divergence, has shown that they experience fewer recombination events (41).

The direction of the $\mathrm{LOH}$ event, i.e. which allele is retained, varies from hybrid to hybrid, or even from niche to niche according to the most advantageous phenotypes

24 (48). Therefore, it is important to understand the genomic alterations that the coexistence of the parental sub-genomes may cause in the hybrid. While in some 
1 hybrids there seems to be a tendency to retain the allele of a given parental lineage, in

2 others this appears to be a random process $(34,40,44,49,50)$. Previous studies have

3 shown that incompatibilities between the mitochondrial and the nuclear genome may

4 be one of the major sources of incompatibilities in hybrids $(51,52)$. Nevertheless, a

5 previous study on Saccharomyces hybrids revealed that the overall direction of LOH

6 is not correlated with the direction of mitochondrial inheritance (53). In M. furfur H1

7 and $\mathrm{H} 2$ hybrid lineages we found that $>60 \%$ of the genome covered by $\mathrm{LOH}$

8 corresponds to the allele of P2, except for the strains CBS6001 and CBS7019 where

9 this value is $45 \%$ (Supplementary Table 2). Although this result may suggest a slight

10 tendency to retain the allele of P2 parental, we consider it to be not sufficiently clear

11 or strong, as in other hybrids where $>80 \%$ of the genome retains the allele of the same

12 parent $(49,50)$. Indeed, if instead of focusing on the percentage of the genome

13 covered by $\mathrm{LOH}$, we analyze the number of $\mathrm{LOH}$ events that favored each of the

14 parental alleles, we see that in three out of the six hybrid strains $\sim 56 \%$ of the events tended to $\mathrm{P} 2$, while in the other three this number is reduced to $43 \%$, thus suggesting high stochasticity in the process.

\section{M. furfur possesses the genetic machinery of a pseudo-bipolar mating system}

To understand the origin of the hybridization events leading to the $\mathrm{H} 1$ and $\mathrm{H} 2$ lineages, analysis of the mating-type genes of $M$. furfur was carried out (see 
1 analyzed are $~ 590 \mathrm{~kb}$ apart (Supplementary Table 3), suggesting a pseudo-bipolar

2 configuration. A representative comparison of the MAT loci of CBS14139 (MAT

$3 \quad a 1 b 1$ ) and CBS7982 (MAT a2b2), as model strains, is shown in Figure 4. The $M$.

4 furfur MAT structure reflects that of the MAT locus of M. yamatoensis $(16,20)$ and

$5 \quad$ differs from the M. globosa and M. sympodialis MAT loci, for which MAT a and MAT

$6 \quad b$ are $\sim 167 \mathrm{~kb}$ and $\sim 140 \mathrm{~kb}$ apart, respectively $(20,22)$. This is consistent with a

$7 \quad$ whole-genome based clustering that groups $M$. furfur and M. yamatoensis in a

8 separate phylogenetic cluster within the Malassezia genus (16). In all M. furfur

9 haploid strains analyzed, the two MAT a locus genes are divergently oriented,

10 corroborating previous findings for other Malassezia species (16, 20, 22). Moreover,

11 a comparison between the MAT $a 1$ and $a 2$ loci revealed that the Mfa and Pra genes

12 have an opposite arrangement albeit being located in highly syntenic flanking regions

13 (Figure 4). In Basidiomycetes, the tetrapolar mating system is thought to be ancestral,

14 and transition from a tetrapolar to bipolar system may be linked to the evolution of

15 pathogenicity $(54,55)$. For example, in the genus Cryptococcus, pathogenic species

16 have a bipolar mating system, whereas closely related non-pathogenic species such as

17 Cryptococcus amylolentus have a tetrapolar mating system (56). Based on findings

18 for the red yeast Sporobolomyces salmonicolor (cited as Sporidiobolus salmonicolor),

19 the pseudo-bipolar mating system was proposed to be a gradual stage in the transition

20 from a tetrapolar to bipolar system (55).

[Figure 4]

24 Hybrid genome searches with the MAT genes of haploid $M$. furfur identified two MAT 
1 and CBS7019 (H2). The sexual identity of the $b$ loci among strains from all lineages

2 was assigned following sequence comparison and phylogenetic analysis of the

3 predicted proteins, resulting in $M A T b 1, b 2, b 3$, and $b 4$ alleles, with MAT b3 and

4 MAT b4 being present only in the hybrids, and closely related to MAT b1 and MAT

5 b2, respectively (Figure 5C). In particular, based on genome data (both genome

6 assembly and read mapping data), strain CBS1878 was designated as MAT a2a2blb4,

7 and strain CBS7019 as MAT ala2b3b4. Interestingly, CBS1878 did not contain MAT

$8 a 1$ but the $a 2$ locus was present in two copies. A closer inspection of the read

9 alignment on the MAT locus with IGV (57) revealed that part of the Illumina paired-

10 end reads flanking this duplication in a 2 had their mate aligning in the edges of the

11 region corresponding to the a1 allele (region present in the reference P1, but without

12 read coverage in $\mathrm{H} 1$ strains), suggesting the occurrence of a $\mathrm{LOH}$ from an ancestral

$13 a 1 a 2$ state to a derived $a 2 a 2$ configuration. Based on read mapping results, a similar

14 occurrence seems to have happened for H1-strains CBS 4172 and CBS9365

15 (Supplementary Figure 1c).

A molecular assay for M. furfur mating type identifies mating-compatible strains in the parental lineages

Following these initial findings for the $M$. furfur mating-type regions, a PCR assay was developed with primers that specifically amplify MAT a1, MAT a2, MAT b1-b3, and MAT $b 2-b 4$. PCR results for the MAT $a 1$ and $a 2$, and sequencing analysis of MAT $b 1-b 3$ and $b 2-b 4$, indicated additional mating-type configurations (Figure 5A-C and Supplementary Table 4). Both P1 and P2 parental lineages contain strains with either MAT a1 or MAT a2, while all P1 strains are of the MAT b2 type and all $\mathrm{P} 2$ strains are of the MAT b1 type (Figure 5A-C and Supplementary Table 4). The presence of $a 1 b 1$, 
$1 \quad a 2 b 1, a 1 b 2$ and $a 2 b 2$ MAT combinations supports the hypothesis that recombination

2 occurs within this region, corroborating findings on the pseudo-bipolar MAT structure

3 of M. sympodialis and in contrast with the lack of recombination reported for Ustilago

4 hordei, which is bipolar (20). Our findings also highlight incompatibility at the B loci

5 within each parental lineage, despite compatibility being present at the MAT a locus,

6 which might explain why sexual reproduction could not be observed under laboratory

7 conditions. Attempts to cross compatible MAT $a$ and $M A T b$ strains belonging to the

$8 \quad$ P1 and P2 parental lineages were also carried out, but also in this case sexual

9 reproduction could not be observed. It seems likely that two individual mating events between representatives of both parental lineages originally resulted in hybrid strains that contained the combination MAT ala2b1b2, yet our data showed that various alterations of the mating loci occurred in the hybrids. All H1-strains lost the MAT al copy and may have duplicated the MAT a2 allele (only confirmed for CBS1878, CBS4172, and CBS9365). Strains belonging to the H2 hybrid lineage still have both parental MAT a copies, with the exception of CBS6093, that seems to have lost the parental MAT a 2 copy. Additionally, two unique MAT $b$ arrangements were observed in the hybrids: MAT $b 4$ in both hybrid lineages, with similarity to MAT b2 of the P1 strains; and MAT b3 which is only present in $\mathrm{H} 2$ strains, and is a phylogenetic sister of MAT b1 of the P2 strains (Figure 5A-C and Supplementary Table 4). Strains of hybrid lineage $\mathrm{H} 1$ retained the MAT bl copy. Considering that recombination in the MAT locus has previously been observed in other hybrid lineages $(40,58,59)$, and is associated with a possible restoration of hybrid fertility $(58,59)$, we hypothesize that strains in lineages $\mathrm{H} 1$ and $\mathrm{H} 2$ may have undergone genomic changes leading to re24 establishment of a viable sexual state. 
$1 \quad$ [Figure 5]

2

3 Targeted sequencing of five nuclear loci and genomic data suggest P1 and P2

$4 \quad$ lineages may be two separate species

$5 \quad$ The species $M$. furfur is represented by two neotype cultures, namely CBS1878 and

6 CBS7019, corresponding to the respective names Malassezia furfur and Pityrosporum

7 ovale (synonym of $M$. furfur), but these belong to the hybrid lineages $\mathrm{H} 1$ and $\mathrm{H} 2$,

8 respectively. Many species are described based on a limited number of nuclear DNA

9 loci, ribosomal loci ITS and LSU being two of the most frequently used taxonomic

markers in fungi, but these loci may not reflect the genetic heterogeneity of a yeast

strain sufficiently as is also shown in this study for the ITS of ribosomal DNA and

three protein coding genes ( $\beta$-tubulin, chitin synthase $(\mathrm{CHS} 2)$, and translation elongation factor 1- $\alpha(E F 1-\alpha)$ ) (Supplementary Figure 3). Phylogenetic analysis of published sequencing data for the intergenic transcribed spacer (IGS) of the rDNA resulted in separate clusters for each of the studied lineages, making it a potential diagnostic tool for identifying hybrids belonging to $\mathrm{H} 1$ or $\mathrm{H} 2$ among genetically uncharacterized M. furfur isolates (Supplementary Figure 3B). As chromatograms of hybrid strains for the protein coding genes possessed multiple sites with two different nucleotide peaks representing both parental backgrounds, they were phased into two sequences representing their respective parental copies (Supplementary Figure 3C-E). Based on ITS data, both hybrid lineages $\mathrm{H} 1$ and $\mathrm{H} 2$ cannot be distinguished from the parental lineage $\mathrm{P} 2$, yet for the most part they contain genetic material from both P1 and P2. Type strains or neotype strains are reference strains, often used as representatives for a species in specific functional assays, but as in this case they represent hybrid lineages with a combined genomic content of two haploid parental 
1 lineages, they may not be the best choice to serve as reference strains and should

2 probably be disqualified to serve as neotypes. At present, all strains considered in this

3 study are classified as M. furfur. However, based on sequence divergence between the

$4 \quad \mathrm{P} 1$ and P2 lineages for the five analyzed nuclear genomic loci it is likely that P1 and

$5 \quad$ P2 may represent two closely related species. For example, ITS sequence similarity

6 between P1 strain CBS9595 and the P2 strain CBS14141 is 98\% but their similarity

7 for protein coding genes is much lower: $97.3 \%$ for $\mathrm{EF} 1-\alpha, 96.4 \%$ for $\beta$-tubulin, and

$8 \quad 87.6 \%$ for $C H S 2$ respectively. According to a study that assessed more than 9000

9 yeast isolates to establish species and genus thresholds for ribosomal ITS and LSU

10 regions, a species-threshold of $98.41 \%$ was presented for ITS (60), which supports the

11 assignment of these lineages into two species. Furthermore, as above-mentioned,

12 based on whole-genome sequence alignments for CBS9595 (P1) and CBS14141 (P2),

13 an overall sequence similarity of $89 \%$ was estimated, further supporting that these

14 strains represent two species.

\section{Hybrid lineages $\mathrm{H} 1$ and $\mathrm{H} 2$ show different morphologies but limited}

\section{differentiation based on traditional physiological properties}

18 At the phenotypic level, cells belonging to hybrid lineages differ in size and shape from parental lineage cells (Figure 6, Supplementary Table 5), with hybrid cells being thinner and more elongated. However, growth profiling experiments traditionally used for Malassezia species identification, seem not to differ significantly between hybrid and parental lineages (Supplementary Table 6). One noteworthy observation is that all P2 strains were positive for $\beta$-glucosidase activity and strains from the other

24 lineages were all negative, except for one P1 strain (CBS9589) and for one H2-strain, 
1 based on mating type, AFLP-pattern, and Matrix-Assisted Laser Desorption

2 Ionization-time Of Flight (MALDI-TOF) mass spectrum (Supplementary Figure 6)

3 and would be an interesting candidate for further genomic exploration. Variable

4 results for Cremophor EL utilization and $\beta$-glucosidase activity have been described

5 previously for $M$. furfur isolates $(12,15,23-26)$ and may be an expression of the

6 heterogeneity of the species. Analysis of mass spectra generated with a Bruker

$7 \quad$ MALDI Biotyper also illustrated the differences between P1 and P2 lineages and

8 formed separate dendrogram clusters for $\mathrm{H} 1, \mathrm{H} 2, \mathrm{P} 1$, and $\mathrm{P} 2$, with a few exceptions

9 (Supplementary Figure 6). The putative H2 strain CBS6093 clustered basal to clusters

10 for $\mathrm{P} 2$ and $\mathrm{H} 2$, supporting above mentioned deviating findings for that strain. In

11 addition, the MALDI-TOF mass spectrum of H1-strain CBS4172 clusters with the P2

12 strains. This divergent character is in line with a deviating PFGE chromosomal

13 banding pattern and this strain has lost MAT a1, and only has one copy of MAT a2.

14 These findings need to be repeated but confirm the current high heterogeneity present

15 in the species M. furfur that might be better interpreted as a species complex.

Based on AFLP, mating type analysis, and Sanger sequencing data, the H1 hybrid lineage consists of seven strains, five originating from animal skin and two from diseased human skin. Hybrid lineage $\mathrm{H} 2$ consists of four strains, all isolated from human diseased skin, with the exception of CBS6093 for which the origin is unknown. Of note, as has also previously been mentioned $(30,61)$, deep-seated isolates (e.g. blood, urine, rectal swabs) seem to almost exclusively belong to the

23 genotype representing the P2 lineage. Such an observation could, at first sight, be at

24 odds with the previously proposed hypothesis that hybridization plays a role in the emergence of pathogenicity (34). However, it is important to note that such a 
1 hypothesis does not imply that hybridization is the only mechanism leading to

2 pathogenicity, and, in the particular case of M. furfur P2 lineage, our results show that

3 it was likely to result from a hybridization event as well. Leong and colleagues

4 explored antifungal susceptibility patterns of 26 M. furfur strains, including some

$5 \quad$ strains also considered in this study (62). Four hybrid strains (H1: CBS1878,

6 CBS7019; H2:CBS6000, CBS600) showed reduced susceptibility to certain azoles.

7 This feature was not exclusive for strains from hybrid lineages $\mathrm{H} 1$ and $\mathrm{H} 2$ but rather

8 seemed linked to disease state backgrounds of strains, and reduced azole

9 susceptibility also included disease isolates belonging to parental lineage P2 (62).

10 Whether hybridization may have facilitated genetic changes driving this reduced

11 azole susceptibility in any of the lineages $\mathrm{H} 1, \mathrm{H} 2$, or $\mathrm{P} 2$, or whether this was the

12 result from mere exposure to these drugs, remains to be elucidated. Considering that

13 five out of the seven H1-strains are derived from animals, we hypothesize that the

14 hybridization event for H1 may have facilitated a host-shift event between humans

15 and animals. This hypothesis should be tested in future analyses including a larger

16 sampling of strains.

18 [Figure 6]

In summary, this study identified two individual hybrid lineages $\mathrm{H} 1$ and $\mathrm{H} 2$, with $\mathrm{P} 1$ and P2 representing their parental lineages, although not the exact parental strains.

We propose that the diploid hybrid lineages $\mathrm{H} 1$ and $\mathrm{H} 2$ are the result of two separate

23 mating events between mating-compatible strains from the P1 and P2 groups.

24 Interestingly, our genome analysis shows that both haploid parental lineages were 
1 their members mated again to form hybrid lineages $\mathrm{H} 1$ and $\mathrm{H} 2$. The various

2 hybridization events and subsequent further evolutionary changes have contributed to

3 additional genetic diversification in M. furfur, but also at the phenotypic level, the

4 hybrid strains differ from their parental lineages. The implications of possible

5 hybridization-driven changes in pathogenicity, or adaptation to new environments,

6 will drive further analysis and in depth examination comparing pathogenicity factors,

7 lipid production and utilization, and salient physiological and phenotypic facets.

$9 \quad$ Materials and Methods (2216 words)

10 DNA extraction

11 To extract DNA for whole-genome sequencing, cells were grown on modified Dixon

12 agar (mDA) (63) for $48 \mathrm{~h}$ at $30^{\circ} \mathrm{C}$ and harvested into $50 \mathrm{ml}$ tubes. Yeast cells were

13 lysed, using the QIAGEN Genomic DNA Purification procedure for yeast samples

14 (Qiagen, Hilden, Germany), with minor modifications. Lyticase incubation was performed for $2 \mathrm{~h}$ at $30^{\circ} \mathrm{C}$, and RNase/Proteinase incubation was performed for $2 \mathrm{~h}$ at $55^{\circ} \mathrm{C}$. Genomic DNA was purified using Genomic-tip 100/G prep columns, according to the manufacturer's handbook. For AFLP, MLST, and the mating type assay, gDNA extraction was performed following the CTAB method as described by (64) with the following modification: DNA was purified in two steps- first with phenol-chloroform and secondly with chloroform only.

AFLP

AFLP analysis was performed according to (65) with some modifications. Various

24 restriction enzymes and adaptor pairs were combined with multiple primer combinations (Supplementary Table 1). A combined restriction ligation reaction was 
1 performed for $2 \mathrm{~h}$ at $37^{\circ} \mathrm{C}$. PCR products were diluted 200x after purification and

2 then combined with Orange600 size standard (Nimagen, Nijmegen, the Netherlands)

3 before fragment analysis on a 3730xl DNA Analyzer (Thermo Fisher Scientific,

4 Waltham, Massachusetts, USA). Data was imported and analyzed using Bionumerics

$5 \quad$ v.7.6.3 (Applied Maths, Sint-Martens-Latem, Belgium), and dendrograms were

6 created with UPGMA clustering and Pearson correlation coefficient. After

7 purification of PCR products, all reactions were diluted 200x.

\section{Whole-genome sequencing}

10 Thirteen samples were selected for whole-genome sequencing data analysis (Table 1).

11 For three of them (CBS7982, CBS14141 and CBS4172), we retrieved Illumina data

12 from SRA (Table 1) (16). For the remaining ones, whole-genome sequencing was

13 performed at the Genomics Unit from the Centre for Genomic Regulation (group 1: specified, the protocol was similar in both groups of strains. Libraries were prepared using the NEBNext Ultra DNA Library Prep kit for Illumina (New England BioLabs, United States) according to manufacturer's instructions. All reagents subsequently mentioned are from the NEBNext Ultra DNA Library Prep kit for Illumina, if not specified otherwise. $1 \mu \mathrm{g}$ of gDNA was fragmented by ultrasonic acoustic energy in Covaris to a size of $\sim 600$ bp in group 1 and $\sim 300-400$ bp in group 2 . After shearing, the ends of the DNA fragments were blunted with the End Prep Enzyme Mix, and

23 then NEBNext Adaptors for Illumina were ligated using the Blunt/TA Ligase Master

24 Mix. The adaptor-ligated DNA was cleaned-up using the MinElute PCR Purification 
1 agarose gel. Size-selected DNA was then purified using the QIAgen Gel Extraction

2 Kit with MinElute columns (Qiagen) and library amplification was performed by

3 PCR with the NEBNext Q5 Hot Start 2X PCR Master Mix and index primers (12-15

4 cycles in group 1, 6 cycles in group 2). A purification step was done using AMPure

$5 \quad$ XP Beads (Agentcourt, United States). The final library was analyzed using Agilent

6 DNA 1000 chip (Agilent) to estimate the quantity and check size distribution, and it

$7 \quad$ was then quantified by qPCR using the KAPA Library Quantification Kit

8 (KapaBiosystems, United States) prior to amplification with Illumina's cBot.

9 Libraries were loaded and sequenced 2 x 125 on Illumina's HiSeq2500 for group 1 and 2 x 101 on Illumina's HiSeq2000 for group 2. Base-calling was performed using

11 Illumina pipeline software. De-convolution was performed using the CASAVA

12 software (Illumina, United States).

Four samples (CBS7982, CBS9595, CBS14141 and CBS1878) were additionally sequenced with PacBio long-read sequencing strategy. In addition, previously generated PacBio sequencing data was used for mating type analysis only, for five samples (CBS14139, CBS8735, PM315, CBS4172, CBS9369 and CBS7019). All samples were sequenced on the PacBio RSII platform (Pacific Biosciences, United States), and, except for CBS9595, libraries were prepared in 2015 with the DNA Template Prep Kit 3.0, and polymerase/template complexes were subsequently formed using Polymerase Binding Kit P6 v2, and then sequenced with Sequencing 
$1 \quad$ P6 v2, and the sample was sequenced using DNA Sequencing Reagent Kit 4.0 v2

2 with 360 min runtime per SMRTcell.

\section{De novo genome assembly}

5 The genomes of the samples exclusively sequenced with PacBio (CBS14139,

6 CBS8735, PM315, CBS4172, CBS9369 and CBS7019) were assembled with HGAP3

7 within the SMRT portal of PacBio, SMRTanalysis v3.1, with standard settings. The

8 genomes of the four samples with short- and long-read sequencing libraries

9 (CBS7982, CBS9595, CBS14141 and CBS1878) were assembled with a pipeline that

10 combines short- and long-read assemblers. Briefly, Illumina reads were filtered and

11 trimmed with Trimmomatic v0.36 (66) and assembled with Platanus v1.2.4 (67).

12 PacBio reads were corrected with Canu (68) and assembled with DBG2OLC

13 (v20180222) (69) using Platanus assembly, MaSurCA v3.3.0 (70), and WTDBG2

The assemblies' quality was assessed with Quast v4.5 (74) and K-mer Analysis

Toolkit v2.4.1 (KAT, (75)). The best assembly for each sample was chosen based on N50, level of fragmentation and estimated assembly completeness by KAT (75). Augustus Web-server $(76,77)$ was used for genome annotation, using Malassezia restricta proteome as training set (accession number: GCA_003290485.1 (78)). mapper web-server using the default settings (80).

\section{Read mapping and variant calling}


$1 \quad$ All paired-end Illumina libraries were inspected with FastQC v0.11.5

2 (http://www.bioinformatics.babraham.ac.uk/projects/fastqc/) and trimmed and filtered

3 with Trimmomatic v0.36 (66). Read mapping was performed with sppIDer pipeline

4 (81) using a combined reference including the genome assembly of CBS9595 (as

5 representative of P1 lineage, Supplementary File 1 for more details) and CBS14141

6 (as representative of P2 lineage, Supplementary File 1 for more details). To guarantee

7 the proper correspondence between the scaffolds of both parentals, we aligned both

8 genomes with the nucmer tool of MUMmer v3 (82). Average genome coverage for

9 each sample was estimated with Samtools v1.9 (83). Variant calling was performed

with HaploTypo v1.0.1 (84) selecting Freebayes v1.3.2 (85) as variant caller, and

using the default settings for the remaining parameters. Read alignment was inspected with the Integrative Genomics Viewer (IGV) (57).

Definition of blocks of high genomic variability

To determine for each of the haplotypes of the hybrid and the parental genomes the regions with high variability when compared to the reference, we used the methodology developed and tested by (40) for LOH blocks definition. Briefly, we used bedtools merge (86) with a distance of $100 \mathrm{bp}$ to merge the homozygous SNPs of each sample, and we set a minimum polymorphic region size of $100 \mathrm{bp}$. These blocks were compared among the different strains using bedtools jaccard (86). The sequence divergence between the reference alleles and the allele of the polymorphic regions was calculated by dividing the number of SNPs overlapping such regions by the total number of base pairs covered by them. Of note, these regions did not

24 represent $\mathrm{LOH}$ regions in the hybrids, as they only reflect the differences with the 
1 reference. Nevertheless, they were used to infer the patterns of the respective parental

2 lineages.

3

\section{$4 \quad$ LOH blocks definition in the hybrid strains}

$5 \quad$ As read mapping of the hybrid strains was performed simultaneously in both parental

6 lineages, it was not possible to define LOH blocks based on the distribution of

7 heterozygous variants, as usually performed (37, 40, 43, 44). Instead, an alternative

8 approach where regions deleted in one parental and duplicated in the alternative one

9 were used as an indicator of recombination, i.e. $\mathrm{LOH}$. Therefore, LOH block

10 definition in hybrid strains relied on read depth of coverage. Briefly, bedtools

11 genomecov (86) was used to determine the number of reads covering each position.

12 Positions covered by 0 reads were considered deleted, while those covered by $150 \%$

13 of the average genome coverage were considered duplicated. Similarly to the

14 procedure developed by (40), we determined a minimum block size of $100 \mathrm{bp}$.

Bedtools intersect was used to determine the intersection of the deleted regions of P1 and the duplicated regions of $\mathrm{P} 2$, and vice-versa. Only duplicated regions in one

17 parent that intersect a deleted region in the alternative one were considered as $\mathrm{LOH}$.

18 An enrichment analysis of the genes overlapping LOH blocks was performed with

19 FatiGO (87).

\section{Mitochondrial genome assembly}

De novo genome assembly of CBS9595 mitochondrial genome was performed with

24 KY911085.1). 


\section{Identification of $M$. furfur mating type region}

$2 \mathrm{P} / \mathrm{R}($ MAT A) and HD (MAT B) loci of M. sympodialis strains ATCC42132 (MAT

$3 \quad a 1 b 1)$ and ATCC44340 (MAT a2b2) (20) were used as query for tBLASTn analysis

4 on the PacBio genomic assemblies of M. furfur haploid strains. The designation of $M$.

5 furfur MAT A loci was assigned following that of the closest $M$. sympodialis orthologs

6 based on the E-value of the tBLASTn outcome, whereas that of MAT B loci was

$7 \quad$ assigned according to phylogenetic clustering of the predicted concatenated HD

8 proteins (see below). In all cases, the $M A T$ genes identified in $M$. furfur strains were

9 confirmed by reciprocal BLASTx on GenBank. The nomenclature of the M. furfur

10 MAT genes follows that of the closely-related Ustilaginomycotina: $m f a$ is the

11 pheromone-encoding gene, pra is the pheromone receptor, $b E$ and $b W$ are the HD

12 transcription factors, followed by a number to distinguish from different alleles (18).

Open reading frames of the $M A T$ genes were predicted by comparison with their MAT genes were aligned with MUSCLE (89) and their phylogenetic reconstruction was performed with MEGA7 (90) using the maximum likelihood method [Tamura 3parameter model with Gamma distribution] and 100 bootstrap replications. Similarly, translated HD proteins were predicted with ExPASy translate tool (91), and concatenated bE-bW sequences were aligned with MUSCLE (89) and the respective 
1 The identified genes of the MAT $a 1, a 2, b 1$, and $b 2$ loci of the $M$. furfur haploid

2 strains were used as queries for BLASTn and tBLASTx analyses to identify the MAT

3 regions in the $M$. furfur hybrid strains CBS1878, CBS4172, and CBS7019. The MAT

$4 \quad a 1$ and MAT a2 designation followed that of the haploid strains used as input. For the

$5 \quad$ identified $M A T B$ loci, the DNA sequences of $b E$ and $b W$ genes and their predicted

6 encoded proteins were aligned with MUSCLE (89) and then subjected to

7 phylogenetic analysis with MEGA7 (90) as described above (data not shown).

\section{Characterization of $M$. furfur mating type}

10 Primers for the amplification of MAT $a 1, a 2, b 1$, and $b 2$ alleles were designed on the

11 basis of an alignment of all MAT loci of the available $M$. furfur parental and hybrid

12 strains derived from their genome sequences. The primers for MAT A loci specifically amplified the $a 1$ or $a 2$ allele, while the primers for the MAT $B$ loci amplified $b 1$ and $b 3$, or $b 2$ and $b 4$ (Supplementary Table 7). For the amplification of the MAT A loci,

ExTaq (Takara) was used following the manufacturer's instructions with the following PCR conditions: initial denaturation $2 \mathrm{~min}$ at $94^{\circ} \mathrm{C}$, followed by 33 cycles of $30 \mathrm{sec}$ at $94^{\circ} \mathrm{C}, 30 \mathrm{sec}$ annealing at $58^{\circ} \mathrm{C}, 1 \mathrm{~min} 15 \mathrm{sec}$ extension at $72^{\circ} \mathrm{C}$, and a final extension of 5 min at $72^{\circ} \mathrm{C}$. For the amplification of the MAT b1-b3, LATaq (Taqara) supplemented with GC Buffer II was used following the manufacturer's instruction, and PCR conditions were: initial denaturation 2 min at $94^{\circ} \mathrm{C}$, followed by 33 cycles of $30 \mathrm{sec}$ at $94^{\circ} \mathrm{C}, 30 \mathrm{sec}$ annealing at $60^{\circ} \mathrm{C}, 2$ min extension at $72^{\circ} \mathrm{C}$, and a final extension of 5 min at $72^{\circ} \mathrm{C}$. The MAT b2-b4 alleles were amplified using the same conditions as reported for MAT b1-b3, except for the use of LATaq (Taqara)

24 supplemented with GC Buffer I. MAT B loci are high in G+C ( 65\%) and the use of specific Taq polymerase was important for their successful amplification. 
2 The amplified MAT b1-b3 and MaT b2-b4 alleles were then sequenced using the

3 primers used for amplification, and sequences were aligned using MEGAX with

4 MUSCLE and then subjected to phylogenetic analysis using the maximum likelihood

5 method [Tamura-Nei model] and 500 bootstrap replications $(89,92)$.

6

$7 \quad$ Scanning Electron Microscopy

8 Hybrid- and parent strains were cultivated on modified Dixon (mDixon) medium for

$9 \quad 72 \mathrm{~h}$ and a loop of cells was suspended in water. The cells were briefly vortexed to

10 dislodge from each other. Droplets of 1,2 and $3 \mu 1$ were gently placed on mDixon

11 agar and dried for $1 \mathrm{~h}$ in a laminar flow cabinet to fix the cells onto the agar. After

12 pre-examination under a stereo microscope, small $4 \times 4 \mathrm{~mm}$ selections with both

13 individual cells and cells grouped together were cut out using a surgical blade

14 (Swann-Morton, no. 11, Sheffield, UK) and glued on a copper sample cup with a

small droplet of frozen-tissue medium (KP-Cryoblock, Klinipath, Duiven, The

Netherlands) and subsequently snap-frozen in nitrogen slush, and transferred into an

Oxford CT1500 Cryostation connected to a JEOL 5600LV scanning electron gold target in the cryostation. Electron micrographs were taken at an acceleration voltage of $5 \mathrm{kV}$.

\section{Data availability}

23 Sequencing data, genome assemblies and annotations are available at NCBI database 24 under the BioProject accessions PRJNA732434 and PRJNA779728. 


\section{Acknowledgements}

2 The authors thank Bart Kraak for some exploratory PCR and microscopy work,

3 Simon Denil for helping with initial bioinformatics assessment of strain CBS1878,

4 Claudia Cafarchia for providing strain CD866 and Marina Marcet-Houben for all the

5 helpful discussions on the bioinformatics analyses. This work was supported by the

6 European Union's Horizon 2020 research and innovation programme under the Marie

7 Sklodowska-Curie grant agreement No H2020-MSCA-ITN-2014-642095. TG group

8 also acknowledges support from the Spanish Ministry of Economy, Industry, and

9 Competitiveness (MEIC) for the EMBL partnership, and grants 'Centro de Excelencia

10 Severo Ochoa 2013-2017' SEV-2012-0208, and BFU2015-67107 co-founded by

11 European Regional Development Fund (ERDF); from the CERCA Programme /

12 Generalitat de Catalunya; from the Catalan Research Agency (AGAUR) SGR857,

13 and grants from the European Union's Horizon 2020 research and innovation

14 programme under the grant agreement ERC-2016-724173. TG also receives support

15 from an INB Grant (PT17/0009/0023 - ISCIII-SGEFI/ERDF). G.I and J.H. were

16 supported by NIH/NIAID R37 award AI39115-24 and R01 award AI50113-16A1.

17 J.H. is fellow and co-director of the CIFAR program Fungal Kingdom: Threats and

18 Opportunities. TD was supported by the A*STAR Industry Alignment Fund

$19(\mathrm{H} 18 / 01 / \mathrm{a} 0 / 016)$. 


\section{References}

2

3 1. Findley K, Oh J, Yang J, Conlan S, Deming C, Meyer JA, Schoenfeld D,

4 Nomicos E, Park M, NIH Intramural Sequencing Center Comparative

$5 \quad$ Sequencing Program, Kong HH, Segre JA. 2013. Topographic diversity of

6 fungal and bacterial communities in human skin. Nature 498:367-370.

7 2. Byrd AL, Belkaid Y, Segre JA. 2018. The human skin microbiome. Nature

8 Reviews Microbiology.

9 3. Soret P, Vandenborght L-E, Francis F, Coron N, Enaud R, Avalos M,

4. Limon JJ, Tang J, Li D, Wolf AJ, Michelsen KS, Funari V, Gargus M, Nguyen

Investigation Group. 2020. Respiratory mycobiome and suggestion of interkingdom network during acute pulmonary exacerbation in cystic fibrosis. Sci Rep 10:3589.

5. Prohic A, Sadikovic TJ, Krupalija-Fazlic M, Kuskunovic-Vlahovljak S. 2016. Malasseziaspecies in healthy skin and in dermatological conditions. International Journal of Dermatology. Yeast Impacting Both Health And Disease. Frontiers Media SA. 
1 7. Iatta R, Cafarchia C, Cuna T, Montagna O, Laforgia N, Gentile O, Rizzo A,

2 Boekhout T, Otranto D, Montagna MT. 2014. Bloodstream infections by

3 Malassezia and Candida species in critical care patients. Medical Mycology.

4 8. Ha CWY, Martin A, Sepich-Poore GD, Shi B, Wang Y, Gouin K, Humphrey G,

$5 \quad$ Sanders K, Ratnayake Y, Chan KSL, Hendrick G, Caldera JR, Arias C,

6 Moskowitz JE, Ho Sui SJ, Yang S, Underhill D, Brady MJ, Knott S, Kaihara K,

7 Steinbaugh MJ, Li H, McGovern DPB, Knight R, Fleshner P, Devkota S. 2020.

8 Translocation of Viable Gut Microbiota to Mesenteric Adipose Drives

$9 \quad$ Formation of Creeping Fat in Humans. Cell.

9. Spatz M, Richard ML. 2020. Overview of the Potential Role of in Gut Health and Disease. Front Cell Infect Microbiol 10:201.

10. Aykut B, Pushalkar S, Chen R, Li Q, Abengozar R, Kim JI, Shadaloey SA, Wu D, Preiss P, Verma N, Guo Y, Saxena A, Vardhan M, Diskin B, Wang W, Leinwand J, Kurz E, Kochen Rossi JA, Hundeyin M, Zambrinis C, Li X, Saxena D, Miller G. 2019. The fungal mycobiome promotes pancreatic oncogenesis via activation of MBL. Nature.

11. Saunte DML, Gaitanis G, Hay RJ. 2020. -Associated Skin Diseases, the Use of Diagnostics and Treatment. Front Cell Infect Microbiol 10:112.

12. Rhimi W, Theelen B, Boekhout T, Otranto D, Cafarchia C. 2020. spp. Yeasts of Emerging Concern in Fungemia. Front Cell Infect Microbiol 10:370.

13. Amend AS, Barshis DJ, Oliver TA. 2012. Coral-associated marine fungi form novel lineages and heterogeneous assemblages. The ISME Journal. 
1 14. Amend A. 2014. From dandruff to deep-sea vents: Malassezia-like fungi are

2 ecologically hyper-diverse. PLoS Pathog 10:e1004277.

15. Theelen B, Cafarchia C, Gaitanis G, Bassukas ID, Boekhout T, Dawson TL. 2018. Malassezia ecology, pathophysiology, and treatment. Medical Mycology.

16. Wu G, Zhao H, Li C, Rajapakse MP, Wong WC, Xu J, Saunders CW, Reeder NL, Reilman RA, Scheynius A, Sun S, Billmyre BR, Li W, Averette AF, Mieczkowski P, Heitman J, Theelen B, Schröder MS, De Sessions PF, Butler G, Maurer-Stroh S, Boekhout T, Nagarajan N, Dawson TL Jr. 2015. Genus-Wide Comparative Genomics of Malassezia Delineates Its Phylogeny, Physiology, and Niche Adaptation on Human Skin. PLoS Genet 11:e1005614.

17. Wang Q-M, Theelen B, Groenewald M, Bai F-Y, Boekhout T. 2014. Moniliellomycetes and Malasseziomycetes, two new classes in Ustilaginomycotina. Persoonia - Molecular Phylogeny and Evolution of Fungi.

18. Fraser JA, Heitman J. 2003. Fungal mating-type loci. Current Biology.

19. Raudaskoski M, Kothe E. 2010. Basidiomycete Mating Type Genes and Pheromone Signaling. Eukaryotic Cell.

20. Gioti A, Nystedt B, Li W, Xu J, Andersson A, Averette AF, Münch K, Wang X, Kappauf C, Kingsbury JM, Kraak B, Walker LA, Johansson HJ, Holm T, Lehtiö J, Stajich JE, Mieczkowski P, Kahmann R, Kennell JC, Cardenas ME, Lundeberg J, Saunders CW, Boekhout T, Dawson TL, Munro CA, de Groot PWJ, Butler G, Heitman J, Scheynius A. 2013. Genomic Insights into the Atopic Eczema-Associated Skin Commensal Yeast Malassezia sympodialis. mBio. 
1 21. Zhu Y, Engström PG, Tellgren-Roth C, Baudo CD, Kennell JC, Sun S, Blake

2 Billmyre R, Schröder MS, Andersson A, Holm T, Sigurgeirsson B, Wu G,

3 Sankaranarayanan SR, Siddharthan R, Sanyal K, Lundeberg J, Nystedt B,

4 Boekhout T, Dawson TL, Heitman J, Scheynius A, Lehtiö J. 2017.

$5 \quad$ Proteogenomics produces comprehensive and highly accurate protein-coding

6 gene annotation in a complete genome assembly ofMalassezia sympodialis.

$7 \quad$ Nucleic Acids Research.

22. Xu J, Saunders CW, Hu P, Grant RA, Boekhout T, Kuramae EE, Kronstad JW, DeAngelis YM, Reeder NL, Johnstone KR, Leland M, Fieno AM, Begley WM, Sun Y, Lacey MP, Chaudhary T, Keough T, Chu L, Sears R, Yuan B, Dawson TL. 2007. Dandruff-associated Malassezia genomes reveal convergent and divergent virulence traits shared with plant and human fungal pathogens. Proceedings of the National Academy of Sciences.

23. Cafarchia C, Latrofa MS, Figueredo LA, da Silva Machado ML, Ferreiro L, Guillot J, Boekhout T, Otranto D. 2011. Physiological and molecular characterization of atypical lipid-dependentMalasseziayeasts from a dog with skin lesions: adaptation to a new host? Medical Mycology.

24. Zinkeviciene A, Norkunas V, Citavicius D. 2012. Atypical non-lipid-dependent strains of Malassezia furfur. Open Life Sciences.

25. González A, Sierra R, Cárdenas ME, Grajales A, Restrepo S, Cepero de García MC, Celis A. 2009. Physiological and molecular characterization of atypical isolates of Malassezia furfur. J Clin Microbiol 47:48-53.

26. Puig L, Rosa Bragulat M, Castellá G, Javier Cabañes F. 2017. Phenotypic and 
1

$2 \quad$ Mycology.

genetic diversity of Malassezia furfur from domestic and zoo animals. Medical

27. Mittag H. 1994. Fine structural investigation ofMalassezia furfur:I. Size and shape of the yeast cells and a consideration of their ploidy. Mycoses.

28. Boekhout T, Kamp M, Guého E. 1998. Molecular typing of Malassezia species with PFGE and RAPD. Med Mycol 36:365-372.

29. Guého E, Midgley G, Guillot J. 1996. The genus Malassezia with description of four new species. Antonie van Leeuwenhoek.

30. Theelen B, Silvestri M, Guého E, van Belkum A, Boekhout T. 2001. Identification and typing of Malassezia yeasts using amplified fragment length polymorphism (AFLP), random amplified polymorphic DNA (RAPD) and denaturing gradient gel electrophoresis (DGGE). FEMS Yeast Res 1:79-86.

31. Theelen B, Batra R, Gupta A, Summerbell R, Boekhout T. Molecular identification of Malassezia species.

32. Peris D, Alexander WG, Fisher KJ, Moriarty RV, Basuino MG, Ubbelohde EJ, Wrobel RL, Hittinger CT. 2020. Synthetic hybrids of six yeast species. Nat Commun 11:2085.

33. López-Fernández H, Bolnick DI. 2007. What causes partial F1 hybrid viability? Incomplete penetrance versus genetic variation. PLoS One 2:e1294.

34. Mixão V, Gabaldón T. 2018. Hybridization and emergence of virulence in opportunistic human yeast pathogens. Yeast. 
1 35. Gabaldón T. 2020. Hybridization and the origin of new yeast lineages. FEMS

$2 \quad$ Yeast Res 20.

3 36. Naranjo $\square$ Ortiz MA, Gabaldón T. 2020. Fungal evolution: cellular, genomic and metabolic complexity. Biological Reviews.

5 37. Mixão V, Gabaldón T. 2020. Genomic evidence for a hybrid origin of the yeast opportunistic pathogen Candida albicans. BMC Biol 18:48.

7 38. Mixão V, Saus E, Boekhout T, Gabaldón T. 2021. Extreme diversification driven by parallel events of massive loss of heterozygosity in the hybrid lineage of Candida albicans. Genetics 217.

39. Mixão V, Hansen AP, Saus E, Boekhout T, Lass-Florl C, Gabaldón T. 2019. Whole-Genome Sequencing of the Opportunistic Yeast Pathogen Candida inconspicua Uncovers Its Hybrid Origin. Frontiers in Genetics.

40. Pryszcz LP, Németh T, Saus E, Ksiezopolska E, Hegedűsová E, Nosek J, Wolfe KH, Gacser A, Gabaldón T. 2015. The Genomic Aftermath of Hybridization in the Opportunistic Pathogen Candida metapsilosis. PLoS Genet 11:e1005626.

41. Priest SJ, Coelho MA, Mixão V, Clancey SA, Xu Y, Sun S, Gabaldón T,

42. Samarasinghe H, Xu J. 2018. Hybrids and hybridization in the Cryptococcus neoformans and Cryptococcus gattii species complexes. Infect Genet Evol $66: 245-255$. 
1 43. Mixão V, Hegedűsová E, Saus E, Pryszcz LP, Cillingová A, Nosek J, Gabaldón

44. Schröder MS, Martinez de San Vicente K, Prandini THR, Hammel S, Higgins DG, Bagagli E, Wolfe KH, Butler G. 2016. Multiple Origins of the Pathogenic Yeast Candida orthopsilosis by Separate Hybridizations between Two Parental Species. PLoS Genet 12:e1006404.

45. Theelen B, Christinaki AC, Dawson TL, Boekhout T, Kouvelis VN. 2021. Comparative analysis of Malassezia furfur mitogenomes and the development of a mitochondria-based typing approach. FEMS Yeast Res 21.

46. Boekhout T, Kurtzman CP, O’Donnell K, Smith MT. 1994. Phylogeny of the yeast genera Hanseniaspora (anamorph Kloeckera), Dekkera (anamorph Brettanomyces), and Eeniella as inferred from partial 26S ribosomal DNA nucleotide sequences. Int J Syst Bacteriol 44:781-786.

47. Boekhout T, Bosboom RW. 1994. Karyotyping of Malassezia Yeasts: Taxonomic and Epidemiological Implications. Systematic and Applied Microbiology.

48. Morales L, Dujon B. 2012. Evolutionary role of interspecies hybridization and genetic exchanges in yeasts. Microbiol Mol Biol Rev 76:721-739.

49. Louis VL, Despons L, Friedrich A, Martin T, Durrens P, Casarégola S, Neuvéglise C, Fairhead C, Marck C, Cruz JA, Straub M-L, Kugler V, Sacerdot C, Uzunov Z, Thierry A, Weiss S, Bleykasten C, De Montigny J, Jacques N, Jung P, Lemaire M, Mallet S, Morel G, Richard G-F, Sarkar A, Savel G, 
1 Schacherer J, Seret M-L, Talla E, Samson G, Jubin C, Poulain J, Vacherie B,

2 Barbe V, Pelletier E, Sherman DJ, Westhof E, Weissenbach J, Baret PV,

3 Wincker P, Gaillardin C, Dujon B, Souciet J-L. 2012. Pichia sorbitophila, an

4 Interspecies Yeast Hybrid, Reveals Early Steps of Genome Resolution After

$5 \quad$ Polyploidization. G3 2:299-311.

6 50. Pontes A, Čadež N, Gonçalves P, Sampaio JP. 2019. A Quasi-Domesticate Relic

$7 \quad$ Hybrid Population of $\times$ Adapted to Olive Brine. Front Genet 10:449.

8 51. Lee H-Y, Chou J-Y, Cheong L, Chang N-H, Yang S-Y, Leu J-Y. 2008.

$9 \quad$ Incompatibility of nuclear and mitochondrial genomes causes hybrid sterility between two yeast species. Cell 135:1065-1073.

52. Jhuang H-Y, Lee H-Y, Leu J-Y. 2017. Mitochondrial-nuclear co-evolution leads to hybrid incompatibility through pentatricopeptide repeat proteins. EMBO Rep 18:87-101.

53. Langdon QK, Peris D, Baker EP, Opulente DA, Nguyen H-V, Bond U, Gonçalves P, Sampaio JP, Libkind D, Hittinger CT. 2019. Fermentation innovation through complex hybridization of wild and domesticated yeasts. Nature Ecology \& Evolution.

54. Bakkeren G, Kämper J, Schirawski J. 2008. Sex in smut fungi: Structure, function and evolution of mating-type complexes. Fungal Genet Biol 45 Suppl $1: \mathrm{S} 15-21$.

55. Coelho MA, Sampaio JP, Gonçalves P. 2010. A Deviation from the BipolarTetrapolar Mating Paradigm in an Early Diverged Basidiomycete. PLoS Genetics. 
1 56. Sun S, Yadav V, Billmyre RB, Cuomo CA, Nowrousian M, Wang L, Souciet J-

2 L, Boekhout T, Porcel B, Wincker P, Granek JA, Sanyal K, Heitman J. 2017.

$3 \quad$ Fungal genome and mating system transitions facilitated by chromosomal translocations involving intercentromeric recombination. PLoS Biol 15:e2002527.

57. Thorvaldsdóttir H, Robinson JT, Mesirov JP. 2013. Integrative Genomics Viewer (IGV): high-performance genomics data visualization and exploration. Brief Bioinform 14:178-192.

58. Watanabe J, Uehara K, Mogi Y, Tsukioka Y. 2017. Mechanism for Restoration of Fertility in Hybrid Zygosaccharomyces rouxii Generated by Interspecies Hybridization. Appl Environ Microbiol 83.

59. Ortiz-Merino RA, Kuanyshev N, Braun-Galleani S, Byrne KP, Porro D, Branduardi P, Wolfe KH. 2017. Evolutionary restoration of fertility in an interspecies hybrid yeast, by whole-genome duplication after a failed matingtype switch. PLOS Biology.

60. Vu D, Groenewald M, Szöke S, Cardinali G, Eberhardt U, Stielow B, de Vries M, Verkleij GJM, Crous PW, Boekhout T, Robert V. 2016. DNA barcoding analysis of more than 9000 yeast isolates contributes to quantitative thresholds for yeast species and genera delimitation. Stud Mycol 85:91-105.

61. Gupta AK, Boekhout T, Theelen B, Summerbell R, Batra R. 2004. Identification and typing of Malassezia species by amplified fragment length polymorphism and sequence analyses of the internal transcribed spacer and large-subunit regions of ribosomal DNA. J Clin Microbiol 42:4253-4260. 
1 62. Leong C, Chan JWK, Lee SM, Lam YI, Goh JPZ, Ianiri G, Dawson TL. 2021.

2 Azole Resistance Mechanisms in Pathogenic Malassezia furfur. Antimicrobial

$3 \quad$ Agents and Chemotherapy.

4 63. Guého-Kellermann E, Boekhout T, Begerow D. 2010. Biodiversity, Phylogeny

$5 \quad$ and Ultrastructure. Malassezia and the Skin.

6 64. O’Donnell K, Cigelnik E, Weber NS, Trappe JM. 1997. Phylogenetic

$7 \quad$ Relationships among Ascomycetous Truffles and the True and False Morels

8 Inferred from 18S and 28S Ribosomal DNA Sequence Analysis. Mycologia.

9 65. Lu X-L, Najafzadeh MJ, Dolatabadi S, Ran Y-P, van den Ende AHGG, Shen Y-

10 N, Li C-Y, Xi L-Y, Hao F, Zhang Q-Q, Li R-Y, Hu Z-M, Lu G-X, Wang J-J,

11 Drogari-Apiranthitou M, Klaassen C, Meis JF, Hagen F, Liu W-D, de Hoog GS.

12 2013. Taxonomy and epidemiology of Mucor irregularis, agent of chronic

13 cutaneous mucormycosis. Persoonia - Molecular Phylogeny and Evolution of

$14 \quad$ Fungi.

66. Bolger AM, Lohse M, Usadel B. 2014. Trimmomatic: a flexible trimmer for Illumina sequence data. Bioinformatics 30:2114-2120.

67. Kajitani R, Toshimoto K, Noguchi H, Toyoda A, Ogura Y, Okuno M, Yabana M, Harada M, Nagayasu E, Maruyama H, Kohara Y, Fujiyama A, Hayashi T, Itoh T. 2014. Efficient de novo assembly of highly heterozygous genomes from whole-genome shotgun short reads. Genome Res 24:1384-1395.

68. Koren S, Walenz BP, Berlin K, Miller JR, Bergman NH, Phillippy AM. 2017. Canu: scalable and accurate long-read assembly via adaptive -mer weighting and repeat separation. Genome Res 27:722-736. 
1 69. Ye C, Hill CM, Wu S, Ruan J, Ma ZS. 2016. DBG2OLC: Efficient Assembly of

2 Large Genomes Using Long Erroneous Reads of the Third Generation

$3 \quad$ Sequencing Technologies. Sci Rep 6:31900.

4 70. Zimin AV, Marçais G, Puiu D, Roberts M, Salzberg SL, Yorke JA. 2013. The

$5 \quad$ MaSuRCA genome assembler. Bioinformatics 29:2669-2677.

6 71. Ruan J, Li H. Fast and accurate long-read assembly with wtdbg2.

7 72. Kolmogorov M, Raney B, Paten B, Pham S. 2014. Ragout-a reference-assisted assembly tool for bacterial genomes. Bioinformatics 30:i302-9.

73. Walker BJ, Abeel T, Shea T, Priest M, Abouelliel A, Sakthikumar S, Cuomo CA, Zeng Q, Wortman J, Young SK, Earl AM. 2014. Pilon: an integrated tool for comprehensive microbial variant detection and genome assembly improvement. PLoS One 9:e112963.

74. Gurevich A, Saveliev V, Vyahhi N, Tesler G. 2013. QUAST: quality assessment tool for genome assemblies. Bioinformatics 29:1072-1075.

75. Mapleson D, Accinelli GG, Kettleborough G, Wright J, Clavijo BJ. 2016. KAT: a K-mer analysis toolkit to quality control NGS datasets and genome assemblies.

76. Hoff KJ, Stanke M. 2013. WebAUGUSTUS--a web service for training AUGUSTUS and predicting genes in eukaryotes. Nucleic Acids Res 41:W1238.

77. Stanke M, Morgenstern B. 2005. AUGUSTUS: a web server for gene prediction in eukaryotes that allows user-defined constraints. Nucleic Acids Research. 
1 78. Park M, Cho Y-J, Lee YW, Jung WH. 2017. Whole genome sequencing analysis

2 of the cutaneous pathogenic yeast Malassezia restricta and identification of the

3 major lipase expressed on the scalp of patients with dandruff. Mycoses 60:188-

5 197.

79. Seppey M, Manni M, Zdobnov EM. 2019. BUSCO: Assessing Genome Assembly and Annotation Completeness. Methods Mol Biol 1962:227-245.

80. Huerta-Cepas J, Forslund K, Coelho LP, Szklarczyk D, Jensen LJ, von Mering C, Bork P. 2017. Fast Genome-Wide Functional Annotation through Orthology Assignment by eggNOG-Mapper. Mol Biol Evol 34:2115-2122.

81. Langdon QK, Peris D, Kyle B, Hittinger CT. 2018. sppIDer: A Species Identification Tool to Investigate Hybrid Genomes with High-Throughput Sequencing. Mol Biol Evol 35:2835-2849.

82. Kurtz S, Phillippy A, Delcher AL, Smoot M, Shumway M, Antonescu C, Salzberg SL. 2004. Versatile and open software for comparing large genomes. Genome Biol 5:R12.

83. Li H, Handsaker B, Wysoker A, Fennell T, Ruan J, Homer N, Marth G, Abecasis G, Durbin R, 1000 Genome Project Data Processing Subgroup. 2009. The Sequence Alignment/Map format and SAMtools. Bioinformatics 25:2078-2079.

84. Pegueroles C, Mixão V, Carreté L, Molina M, Gabaldón T. 2020. HaploTypo: a variant-calling pipeline for phased genomes. Bioinformatics 36:2569-2571.

85. Garrison E, Marth G. 2012. Haplotype-based variant detection from short-read sequencing. arXiv 1207.3907. 
1 86. Quinlan AR, Hall IM. 2010. BEDTools: a flexible suite of utilities for comparing genomic features. Bioinformatics 26:841-842.

87. Al-Shahrour F, Díaz-Uriarte R, Dopazo J. 2004. FatiGO: a web tool for finding significant associations of Gene Ontology terms with groups of genes. Bioinformatics 20:578-580.

88. Dierckxsens N, Mardulyn P, Smits G. 2017. NOVOPlasty: de novo assembly of organelle genomes from whole genome data. Nucleic Acids Res 45:e18.

89. Edgar RC. 2004. MUSCLE: multiple sequence alignment with high accuracy and high throughput. Nucleic Acids Res 32:1792-1797.

90. Kumar S, Stecher G, Tamura K. 2016. MEGA7: Molecular Evolutionary Genetics Analysis Version 7.0 for Bigger Datasets. Molecular Biology and

91. Gasteiger E, Gattiker A, Hoogland C, Ivanyi I, Appel RD, Bairoch A. 2003.

92. Kumar S, Stecher G, Li M, Knyaz C, Tamura K. 2018. MEGA X: Molecular Evolutionary Genetics Analysis across Computing Platforms. Mol Biol Evol $35: 1547-1549$. 


\section{Tables}

Table 1. Strains used in this study with information about their lineage, source, country of isolation, NGS sequencing data analysis, usage for

PFGE, AFLP, sanger sequencing, mating-type, FACS, LM, MALDI-TOF and physiology analyses.

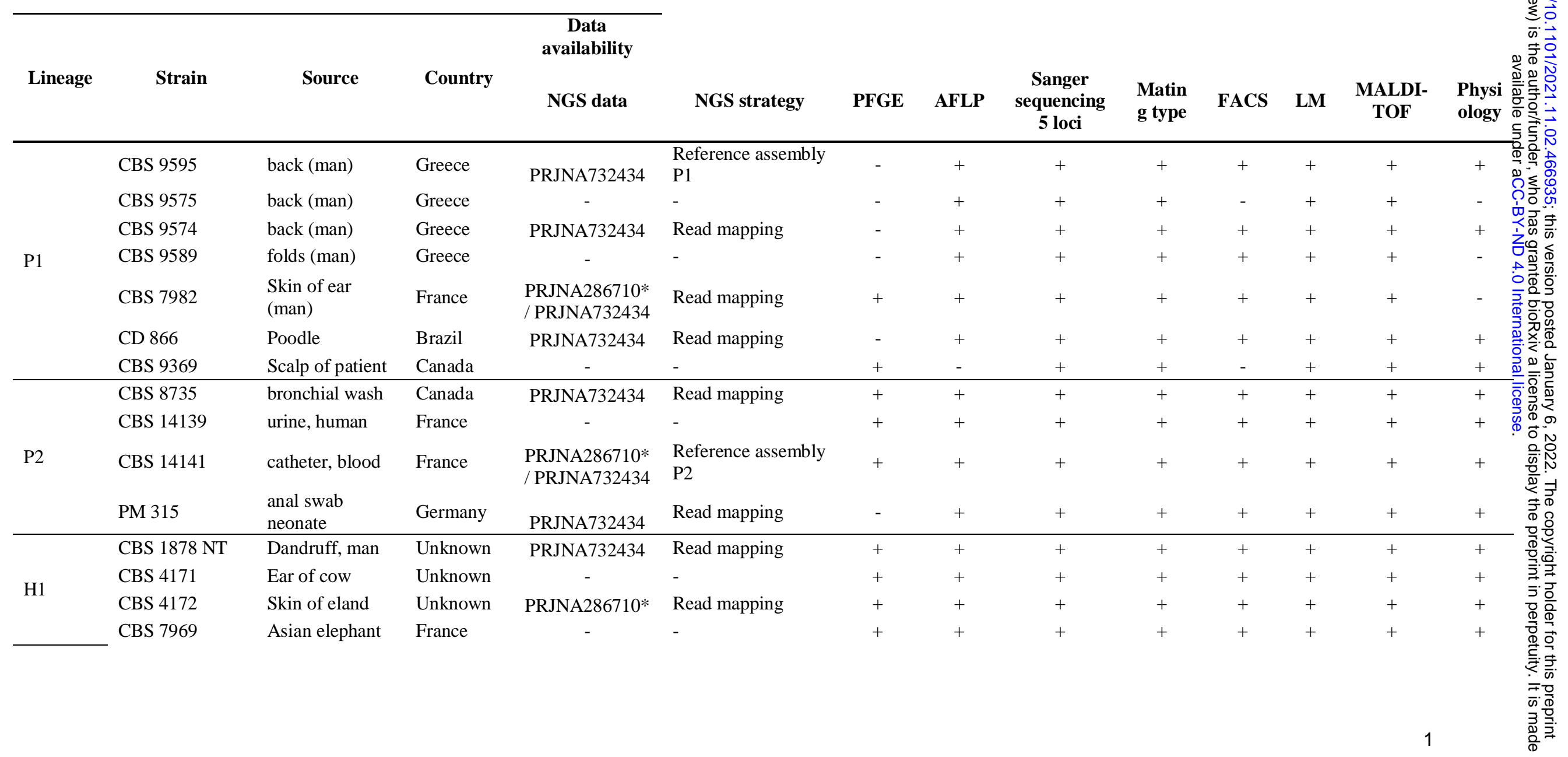




\begin{tabular}{|c|c|c|c|c|c|c|c|c|c|c|c|c|c|}
\hline & CBS 9365 & Elephant in zoo & France & PRJNA732434 & Read mapping & + & + & + & + & + & + & + & + \\
\hline & CBS 5101 & $\begin{array}{l}\text { skin scales, } \\
\text { from PV }\end{array}$ & USA & - & - & - & + & + & + & - & + & + & - \\
\hline & CBS 4162 & Ear of pig & unknown & - & - & - & + & + & + & - & + & + & - \\
\hline \multirow{4}{*}{$\mathrm{H} 2$} & CBS 6000 & Dandruff & India & PRJNA732434 & Read mapping & + & + & + & + & + & + & + & + \\
\hline & CBS 6001 & PV & India & PRJNA732434 & Read mapping & + & + & + & + & - & + & + & - \\
\hline & CBS 6093 & Unknown & unknown & - & - & - & + & + & + & - & + & + & - \\
\hline & CBS 7019 NT & $\begin{array}{l}\text { PV on trunk of } \\
15 \text {-year-old girl }\end{array}$ & Finland & PRJNA732434 & Read mapping & + & + & + & + & + & + & + & + \\
\hline
\end{tabular}

H1 = hybrid lineage 1, H2 = hybrid lineage 2, P1 = Parental lineage 1, P2 = parental lineage 2, PFGE = Pulsed-Field Gel Electrophoresis, AFLP

= Amplified Fragment Length Polymorphism. NT = neotype strain, $+=$ analysis performed, $-=$ analysis not performed, $*$ Data from Wu et al. 2015. 
Table 2. Summary of assembly statistics for the best genome assembly obtained for CBS9595 and CBS14141.

\begin{tabular}{lcc}
\hline & CBS9595 & CBS14141 \\
\hline Assembly strategy & Ragout & DBG2OLC_sparse \\
Estimated genome size (Mb)* & 7.8 & 8.3 \\
Genome size (bp) & 8107776 & 8241990 \\
Contigs & 8 & 9 \\
Contigs > 50kb & 8 & 8 \\
N50 (bp) & 1622862 & 1642932 \\
GC (\%) & $64,48 \%$ & $64,97 \%$ \\
Mapped reads (\%) & $95,50 \%$ & $77,2 \% * * *$ \\
Protein-coding genes & 4.376 & 4.441 \\
Completeness (BUSCO)* & $96.1 \%$ & $95.4 \%$ \\
Completeness (KAT)** & $99,90 \%$ & $99,81 \%$ \\
\hline *BUSCO (79),**KAT (75) & &
\end{tabular}

*BUSCO (79), **KAT (75)

\section{Figures}



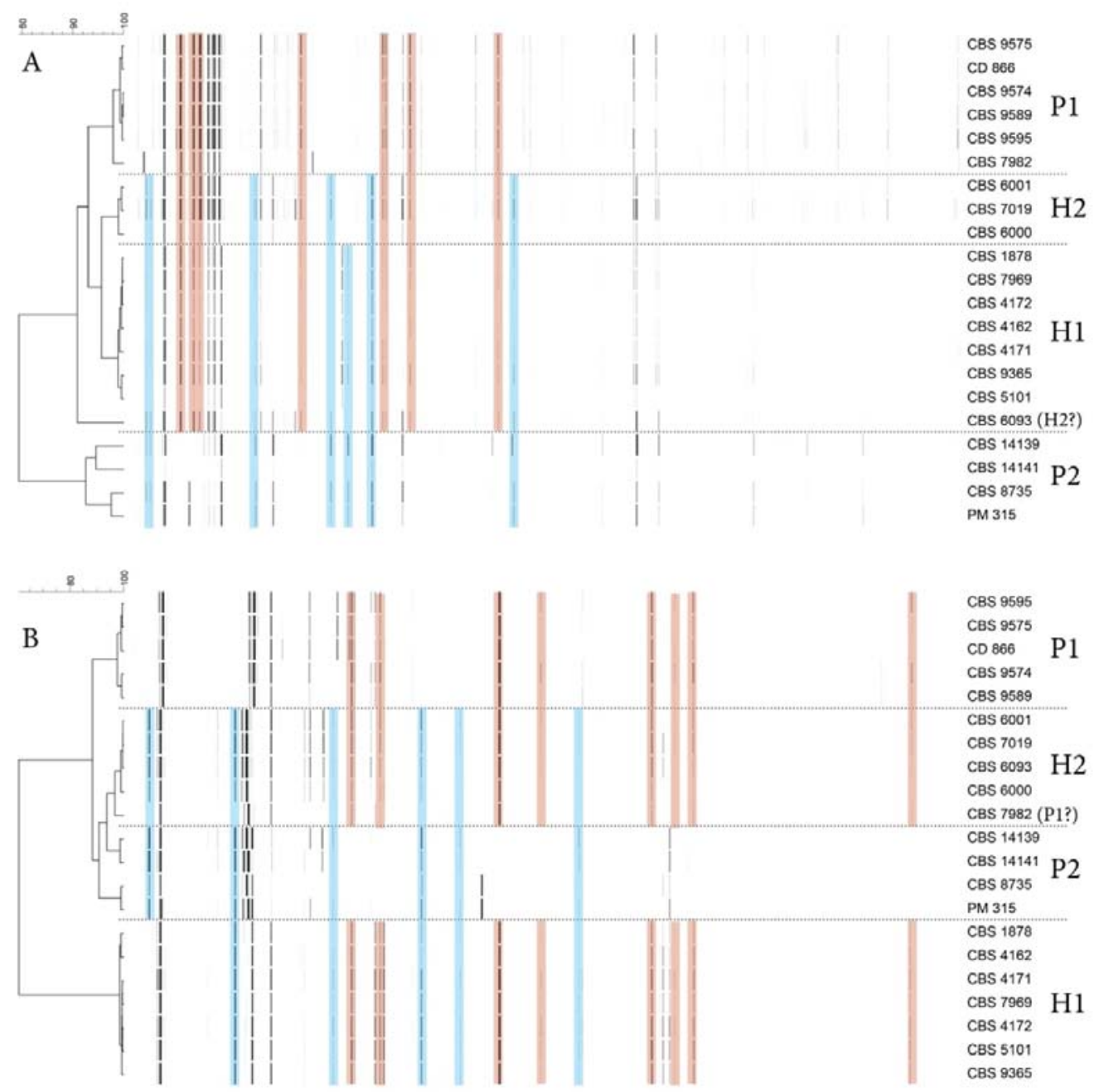

Figure 1. AFLP banding pattern representations derived from electropherograms with Neighbor Joining trees for two different adaptor/primer combinations (A-B) (see Materials \& Methods, and Supplementary Table 1). The horizontal scale represents the similarity percentage. Pink shading highlights restriction fragments shared between parental 1 lineage and hybrids; blue shading highlights shared restriction fragments between parental lineage 2 and hybrids. Both versions resulted from using different primer/adaptor pairs, reflecting different polymorphisms in the genomic DNA and thus resulting in some clustering variation for some strains. CBS6093 belongs to the $\mathrm{H} 2$ lineage based on dendrogram $\mathrm{B}$, but clusters outside any of the other lineages in dendrogram A, suggesting genomic deviation from other $\mathrm{H} 2$ strains, 
a finding also supported by mating type, $\beta$-glucosidase activity, and MALDI-TOF

data. CBS7982 clusters together with other P1 strains as expected in dendrogram A,

but clusters close to H2 strains in dendrogram B. Interestingly, CBS7982 was found

to contain a mitochondrial sequence different from other P1 strains (CBS9595,

CD866, and CBS9574, Supplementary Figure 1).

A
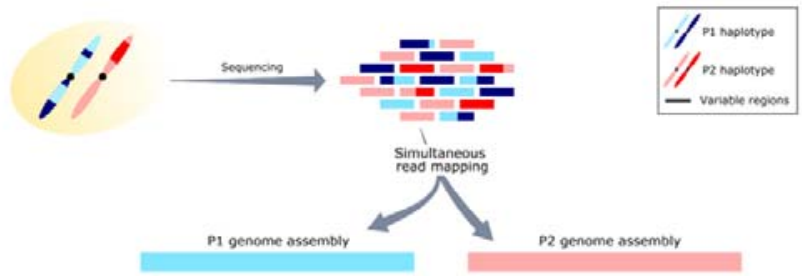

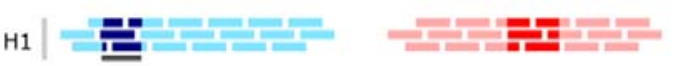

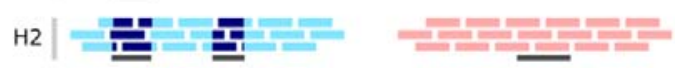

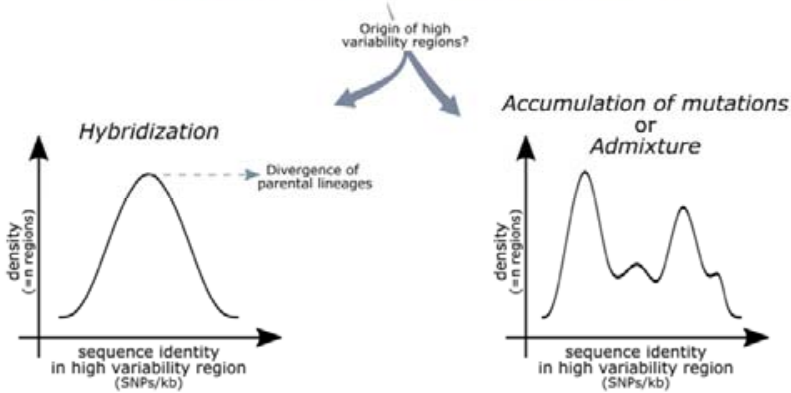

B

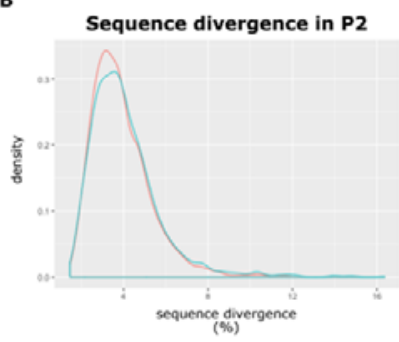

c

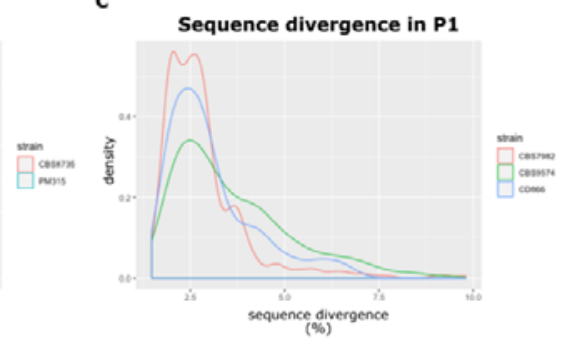

Figure 2. Analysis of the genomic patterns of hybrid genomes. A) Hybrid genomes were sequenced originating sequencing reads from P1 (blue rectangles) and P2 (pink rectangles). These reads were simultaneously aligned to the combined reference of P1 and P2. Light blue and light pink correspond to the alleles present in this reference. Dark blue and dark pink correspond to alleles which are aligned in P1 or P2, 
respectively, but present lower sequence identity forming blocks of genomic variability. Differences in the patterns of genomic variability were used to determine the different hybrid lineages. Estimated sequence divergence between the two alleles (i.e. between dark blue and light blue, or between dark pink and light pink) in terms of SNPs/bp in the blocks of genomic variability were used to determine the origin of such blocks: hybridization or admixture between different strains. B) Sequence divergence in the blocks of genomic variability of $\mathrm{P} 2$ lineages show a single density peak, suggesting a hybrid origin. C) Sequence divergence in the blocks of genomic variability of $\mathrm{P} 1$ lineages show multiple density peaks, with a single peak shared by all strains, not allowing the exclusion of any of the above-mentioned scenarios.

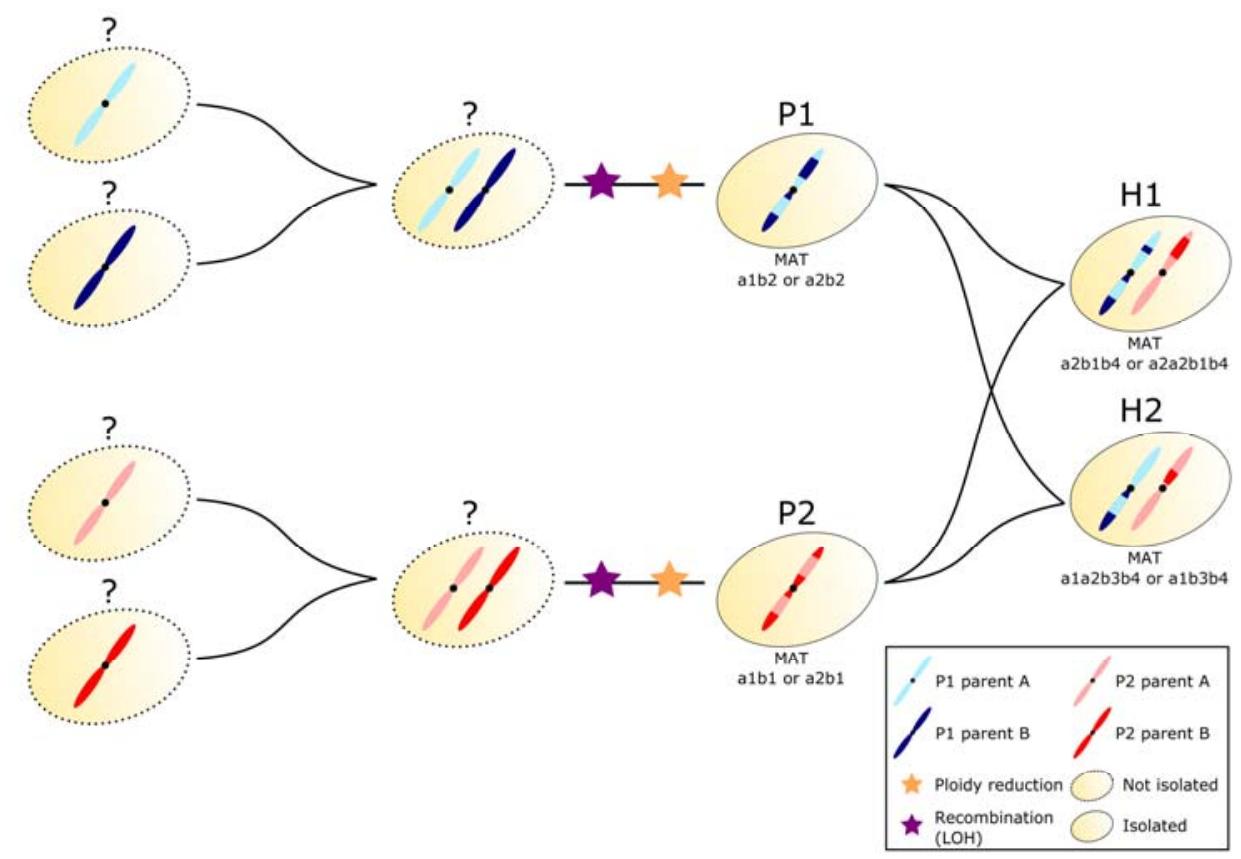

Figure 3. Comparative genomics analysis suggests multiple hybridization events in the evolutionary path of $M$. furfur lineages. 


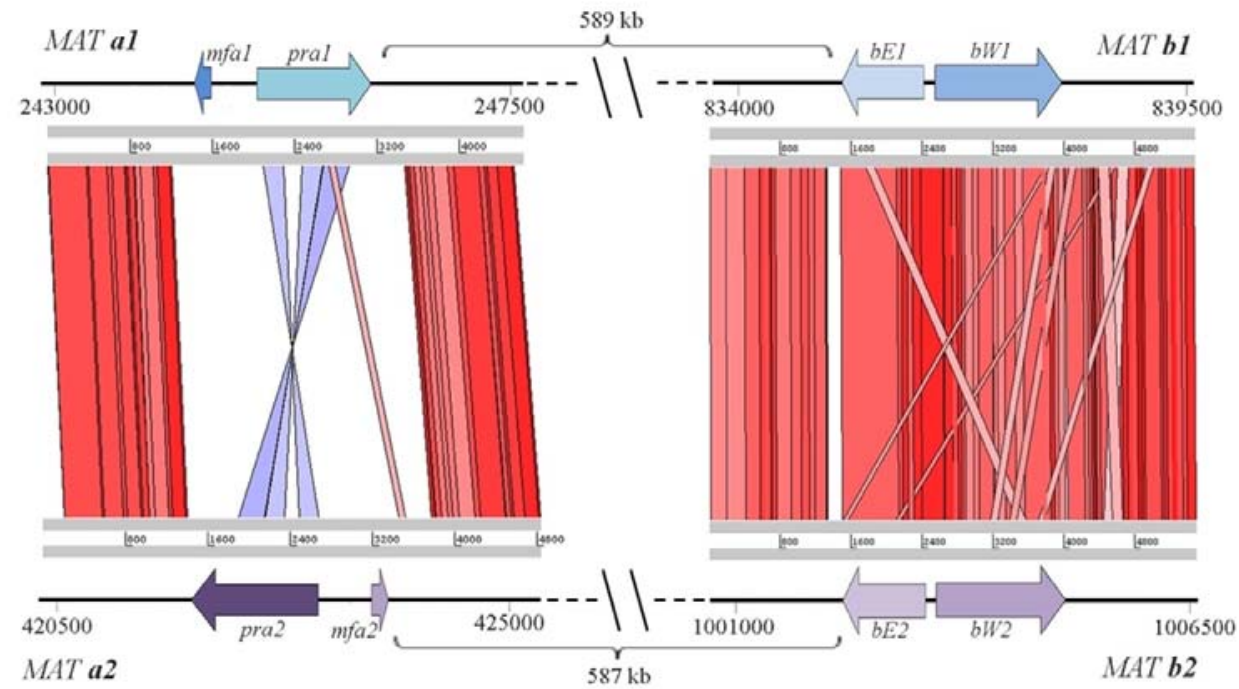

Figure 4. Schematic representation and comparison of mating loci for two mating compatible parental strains: MAT a1b1 loci of M. furfur strain CBS14139 (P2), and of the MAT a2b2 loci of M. furfur strain CBS7982 (P1). The coordinates of the genes in the genome scaffold are indicated. The two MAT regions were aligned with tBLASTx and visualized using ACT Artemis. The red and blue bars indicate regions of similarity, with red bars corresponding to regions of similar orientation and blue bars indicating regions oriented in opposite directions. 
A

MAT a1

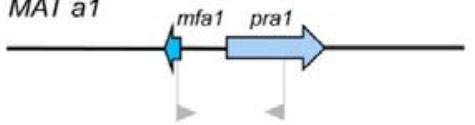

MAT a2

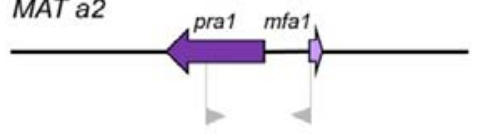

MAT b1-b3

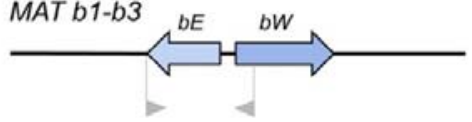

MAT b2-b4

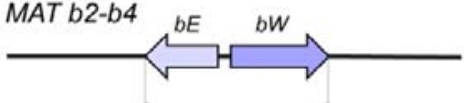

C

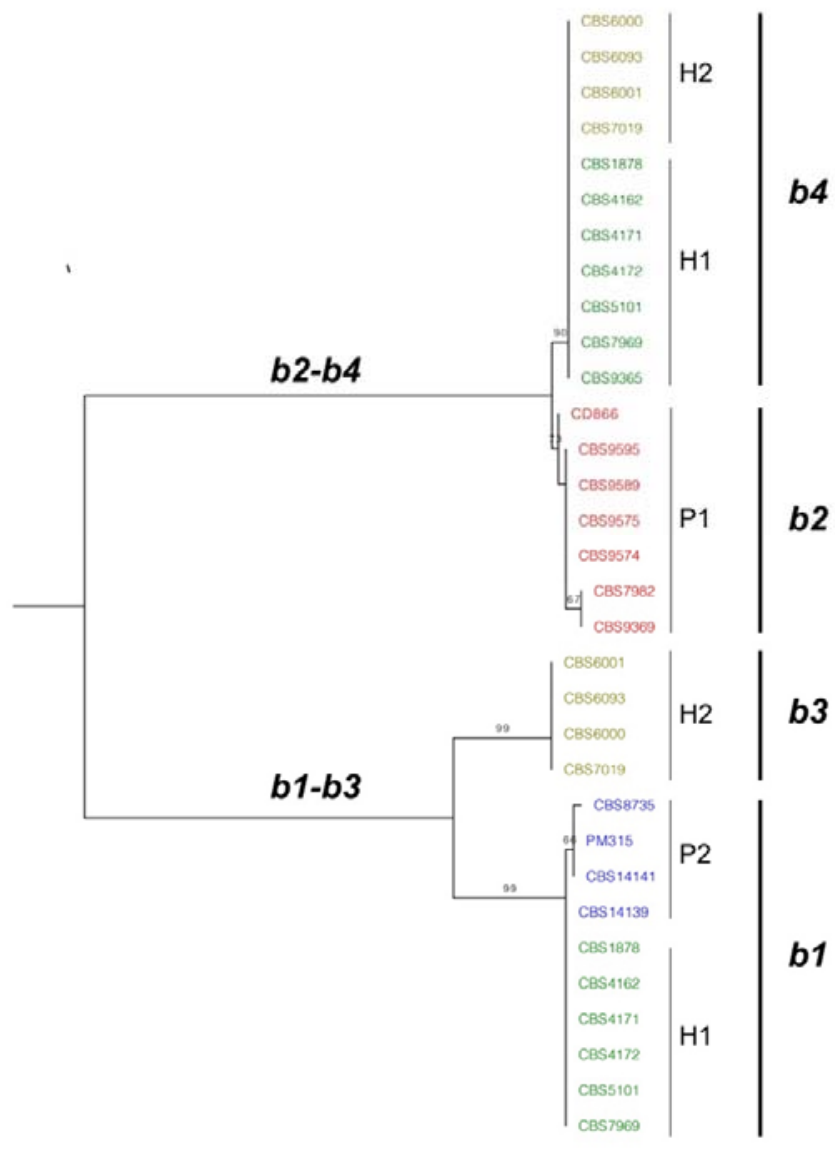

B

C

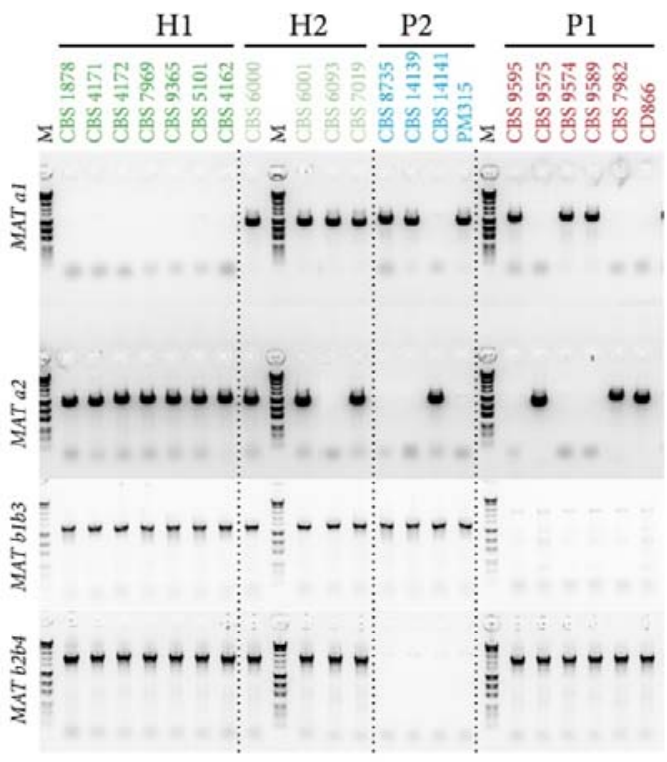

Figure 5. Mating typing assay results. A) Schematic presentation of primer positions in the MAT genes. B) Agarose gel electrophoresis picture, showing PCR results for 
all assessed strains for Mat a1, MAT a2, combined MAT b1-b3, and MAT b2-b4. To distinguish between $\mathrm{b} 1$ and b3, and between $\mathrm{b} 2$ and b4, the MAT B PCR-positive products require sequencing. C) Phylogenetic tree based on the Maximum Likelihood method and Tamura-Nei model with 500 bootstrap replications, representing the MAT B loci, resulting in four main clusters.

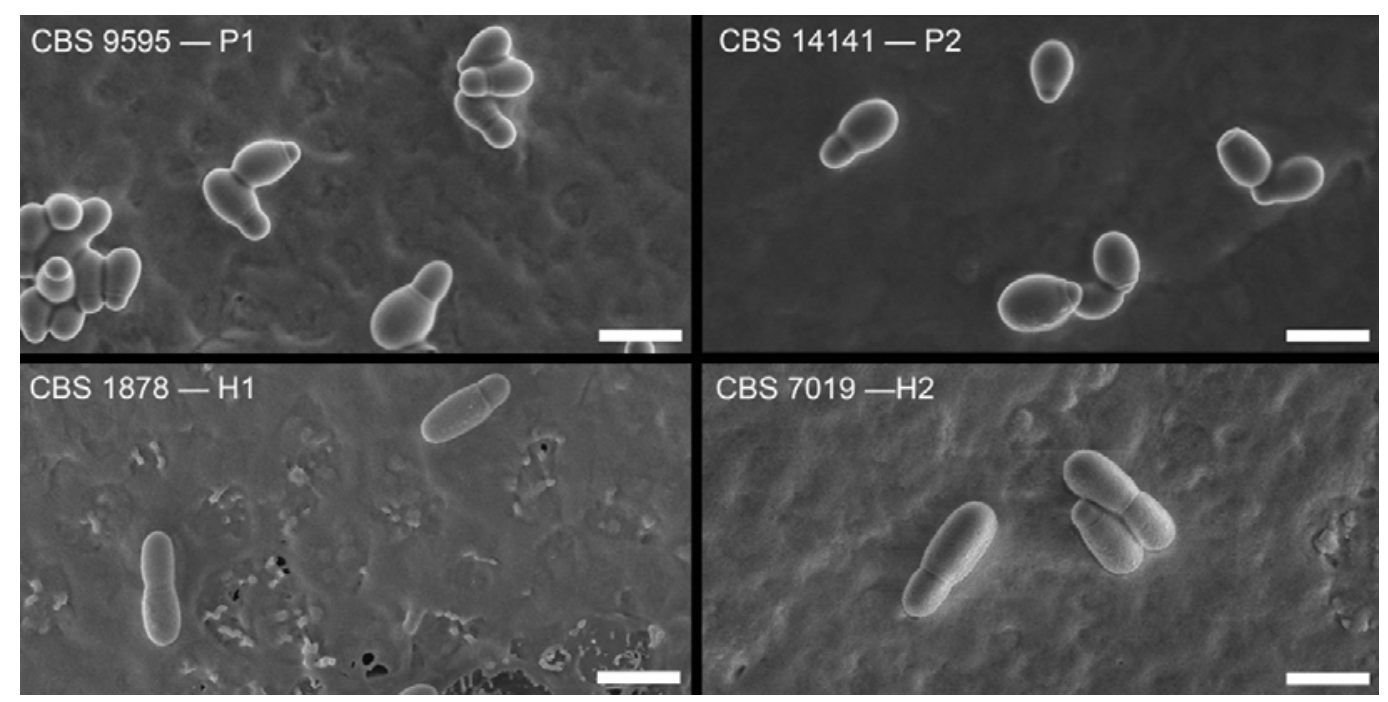

Figure 6. SEM photo plate for representative strains of each lineage, showcasing different morphologies between haploid parental strains and hybrid strains. Additional phenotypic data is available in Supplementary tables 8 and 9 for cell size measurements and traditional physiological data respectively. Bar $=51 \mathrm{~m}$

\section{Supplementary material}

Supplementary Table 1. Adaptor, primer sequences and PCR conditions for AFLP analysis.

Supplementary Table 2. Summary metrics of the whole-genome sequencing analyses of M. furfur strains. 
Supplementary Table 3. tBLASTn E-values for the identification of M. furfur mating type analysis.

Supplementary Table 4. Mating type PCR amplification results for studied strains.

Supplementary Table 5. Cell measurements with light microscopy after $72 \mathrm{~h}$ at $33^{\circ} \mathrm{C}$ incubation on mDixon medium.

Supplementary Table 6. Temperature growth profiles and physiological characteristics of M. furfur strains. Growth at $6^{\circ} \mathrm{C}, 15^{\circ} \mathrm{C}, 24^{\circ} \mathrm{C}, 30^{\circ} \mathrm{C}, 33^{\circ} \mathrm{C}, 40^{\circ} \mathrm{C}$, and $42^{\circ} \mathrm{C}$ on mDA (modified Dixon Agar), SGA (Sabouraud glucose agar), and YNBA (nitrogen base agar); Catalase activity; $\beta$-glucosidase activity; Tween diffusion test on SGA and $\mathrm{YNBA}$ at $15^{\circ} \mathrm{C}, 33^{\circ} \mathrm{C}$, and $37^{\circ} \mathrm{C}$ for selected strains with Tween 20, 40, 60, 80 and CrEL (Cremophor EL).

Supplementary Table 7. Primer sequences for the MAT locus analysis.

Supplementary File 1. Genome assembly of $M$. furfur parental lineages and comparison with the available genomes at public databases.

Supplementary File 2. Additional details regarding analysis of the mating type system of $M$. furfur.

Supplementary Figure 1. IGV screenshots showing the genomic patterns of M. furfur. A) Read mapping of CBS1878 and CBS7019 on the reference genome, 
showing an example of a region of high genomic variability. B) Read mapping of P1 strains to CBS7982 mitochondrial genome assembly. C) Read mapping of H1 strains to MAT a2 loci of P2, showing the occurrence of a duplication.

Supplementary Figure 2. A) Karyotype variation (PFGE) and B) FACS analysis present variation between both hybrid lineages $\mathrm{H} 1$ and $\mathrm{H} 2$ at the chromosomal and ploidy levels. PFGE gels were run under different conditions (see supplementary materials and methods and supplementary Table 2).

Supplementary Figure 3. Phylogenetic trees for five nuclear DNA loci.

Phylogenetic trees based on the Maximum Likelihood method and Tamura-Nei model with 500 bootstrap replications for A) internal transcribed spacer (ITS) and B) the intergenic spacer 1 region (IGS1) of the ribosomal DNA. The IGS1 tree illustrates the suitability of this locus for discrimination between the four studied lineages. The ITS tree showcases the lack of discrimination between both hybrid lineages and the P2 lineage. Additional phylogenetic trees are presented for protein coding genes C) $\beta$ tubulin, D) TEF1 and E) CHS2, illustrating the potential difficulties when sequencing protein coding genes for hybrid strains as at multiple spots in the chromatograms, two different base peaks are present, representing both parental haplotypes. Therefore, the sequences of the protein coding were phased into two copies, based on their parental haplotypes.

Supplementary Figure 4. Coverage plot obtained from sppIDer pipeline (81) for the H1 strains used for whole-genome sequencing data analysis when aligned to a 
combined reference genome of CBS9595 (P1 lineage, red) and CBS14141 (P2

lineage, blue). Dashed lines divide the different chromosomes.

Supplementary Figure 5. Coverage plot obtained from sppIDer pipeline (81) for the

$\mathrm{H} 2$ strains used for whole-genome sequencing data analysis when aligned to a

combined reference genome of CBS9595 (P1 lineage, red) and CBS14141 (P2

lineage, blue). Dashed lines divide the different chromosomes.

Supplementary Figure 6. Dendrogram based on MALDI-TOF MS generated Main Spectra (MSPs) as a measure of general phenotypic variation at the proteomic level, illustrating variation between the various lineages

Supplementary Materials and Methods. Descriptions of Pulsed-Field Gel

Electrophoresis (PFGE), Fluorescence-activated cell sorting (FACS), Matrix-Assisted Laser Desorption Ionization-time Of Flight Mass Spectrometry (MALDI-TOF MS), Microscopy (Light microscopy - cell size measurements), Physiology, and Sanger sequencing and phylogenetic analysis. 